\title{
Synthesis, Cytotoxic and Anti-proliferative Activity of Novel Thiophene, Thieno[2,3-b]pyridine and Pyran Derivatives Derived from 4,5,6,7-tetrahydrobenzo $[b]$ thiophene Derivative
}

\author{
Rafat Milad Mohareb, ${ }^{1, *}$ Nadia Youssef Megally Abdo \\ and Fatma Omar Al-farouk ${ }^{3}$ \\ ${ }^{1}$ Department of Chemistry, Faculty of Science, Cairo University, Giza, A. R. Egypt \\ ${ }^{2}$ Chemistry Department, Faculty of Education, Alexandria University, 21526 Alexandria, Egypt \\ ${ }^{3}$ Department of Chemistry, Faculty of Science, American University in Cairo, 5th Settlement, A.R., Egypt \\ *Corresponding author: E-mail: raafat_mohareb@yahoo.com
}

Received: 15-09-2016

\begin{abstract}
Novel tetrahydrobenzo[b]thienopyrole derivatives are synthesized from 2-amino-3-cyano-4,5,6,7-tetrahydrobenzo[b]thiophene (1) through its reaction with $\alpha$-chloroacetone to give the corresponding $N$-alkyl derivative 3 . Compound 3 undergoes ready cyclization in sodium ethoxide solution to give the tetrahydrobenzo[b]thienopyrrole 4 . The latter compound 4 is used as the key starting material for the synthesis of thiophene, thieno[2,3-b]pyridine and pyran derivatives. The cytotoxicity of the synthesized products towards the human cancer cell lines namely gastric cancer (NUGC), colon cancer (DLD-1), liver cancer (HA22T and HEPG-2), breast cancer (MCF-7), nasopharyngeal carcinoma (HONE-1) and normal fibroblast (WI-38) cell lines are measured. Compounds 4, 7a, 7b, 8a, 8b, 10c, 10d, 10f, 12a, 12b, 14b and 15b exhibit the optimal cytotoxic effect against cancer cell lines. Compounds $\mathbf{7 b}$ and $\mathbf{1 4 b}$ show the maximum inhibitory effect and these are much higher than the reference CHS-828 (pyridyl cyanoguanidine). On the other hand, the anti-proliferative evaluations of these compounds with high potency against the cancer cell lines L1210, Molt4/C8, CEM, K562, K562/4 and HCT116 show that compounds $\mathbf{7 b}$ and $\mathbf{8 b}$ give $\mathrm{IC}_{50}$ 's against Molt4/C8 and CEM cell lines higher than that of the reference, doxorubicin.
\end{abstract}

Keywords: Tetrahydrobenzo[b]thiophene, pyran, thiophene, cytotoxicity, anti-proliferative activity

\section{Introduction}

Sulfur containing heterocycles paved way for the active research in the pharmaceutical Chemistry. Nowadays benzothiophene derivatives in combination with other ring systems have been used extensively in pharmaceutical applications. ${ }^{1-3} \mathrm{~A}$ large number of compounds containing thiophene system have been investigated because of their broad spectrum of biological activities which include analgesic, ${ }^{4}$ antibacterial,${ }^{5}$ antifungal,${ }^{6}$ antiparasitic, ${ }^{7}$ antiviral,${ }^{8}$ anti-inflammatory, ${ }^{9}$ anticonvulsant, ${ }^{10}$ anti-nociceptive, ${ }^{11}$ DNA cleavage,${ }^{12}$ herbicidal, ${ }^{13}$ antitubercular, ${ }^{14}$ protein kinase inhibition, ${ }^{15}$ respiratory syndrome protease inactivation, ${ }^{16}$ an active ester in the peptide synthesis and agonists of peroxisome proliferator activated receptors. ${ }^{17}$ In addition to these considerable biological applications, tetrahydrobenzo $[b]$ thiophenes are important intermediates, protecting groups and final products in organic synthesis. Recently, our research group was involved through comprehensive program aiming for the synthesis of 4,5,6,7-tetrahydroben$\mathrm{zo}[b]$ thiophene derivatives followed by their antitumor evaluations. ${ }^{18,19}$ Moreover, we reported the multi-component reactions with $3-(\alpha$-bromoacetyl)coumarin to give pyan and pyrididine derivatives. ${ }^{20}$ In continuation of this program we are demonstrating the use of 2-amino-3-cyano4,5,6,7-tetrahydrobenzo[b]thiophene for the synthesis of tetrahydrobenzo $[b]$ thienopyrrole derivatives followed by their cytotoxic and the anti-proliferative evaluations. ${ }^{21,22}$ 


\section{Results and Discussion}

The reaction of the 2-amino-3-cyano-4,5,6,7-tetrahydrobenzo[ $[b]$ thiophene $(\mathbf{1})$ with $\alpha$-chloroacetone in the presence potassium carbonate afforded the 2-((2-oxopropyl)amino)-4,5,6,7-tetrahydrobenzo[ $b]$ thiophene-3carbonitrile (3). Compound $\mathbf{3}$ was characterized by ${ }^{1} \mathrm{H}-\mathrm{NMR}$ and ${ }^{13} \mathrm{C}-\mathrm{NMR}$. Thus, the ${ }^{1} \mathrm{H}-\mathrm{NMR}$ spectrum display the presence of beside the expected tetrahydrobenzene moiety, a singlet at $\delta 5.20 \mathrm{ppm}$ indicating the presence of the $\mathrm{N}-\mathrm{CH}_{2}$ group, a singlet at $\delta 2.88 \mathrm{ppm}$ assigned to the $\mathrm{CH}_{3}$ group and a broad singlet at $\delta 8.30$ $\mathrm{ppm}$ due to the NH group. Moreover, the ${ }^{13} \mathrm{C}$-NMR spectrum showed $\delta$ : $19.6\left(\mathrm{CH}_{3}\right), 20.3,22.0,25.7$ and 34.6 (4 $\left.\mathrm{CH}_{2}\right), 55.6\left(\mathrm{CH}_{2}\right), 116.8(\mathrm{CN}), 124.1,124.9,128.7$ and 139.5 (thiophene $\mathrm{C}), 164.8(\mathrm{C}=\mathrm{O})$. Compound 3 under-<smiles>N#Cc1c(N)sc2c1CCCC2</smiles>

1<smiles></smiles>

2<smiles>CC#CC[C@H]1CCc2c(sc(NCC(C)=O)c2C#N)C1</smiles>

3

3

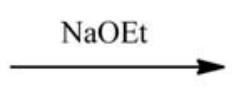<smiles>CC(=O)c1[nH]c2sc3c(c2c1N)CCCC3</smiles>

4

Shema 1. Synthesis of compounds 3 and 4.

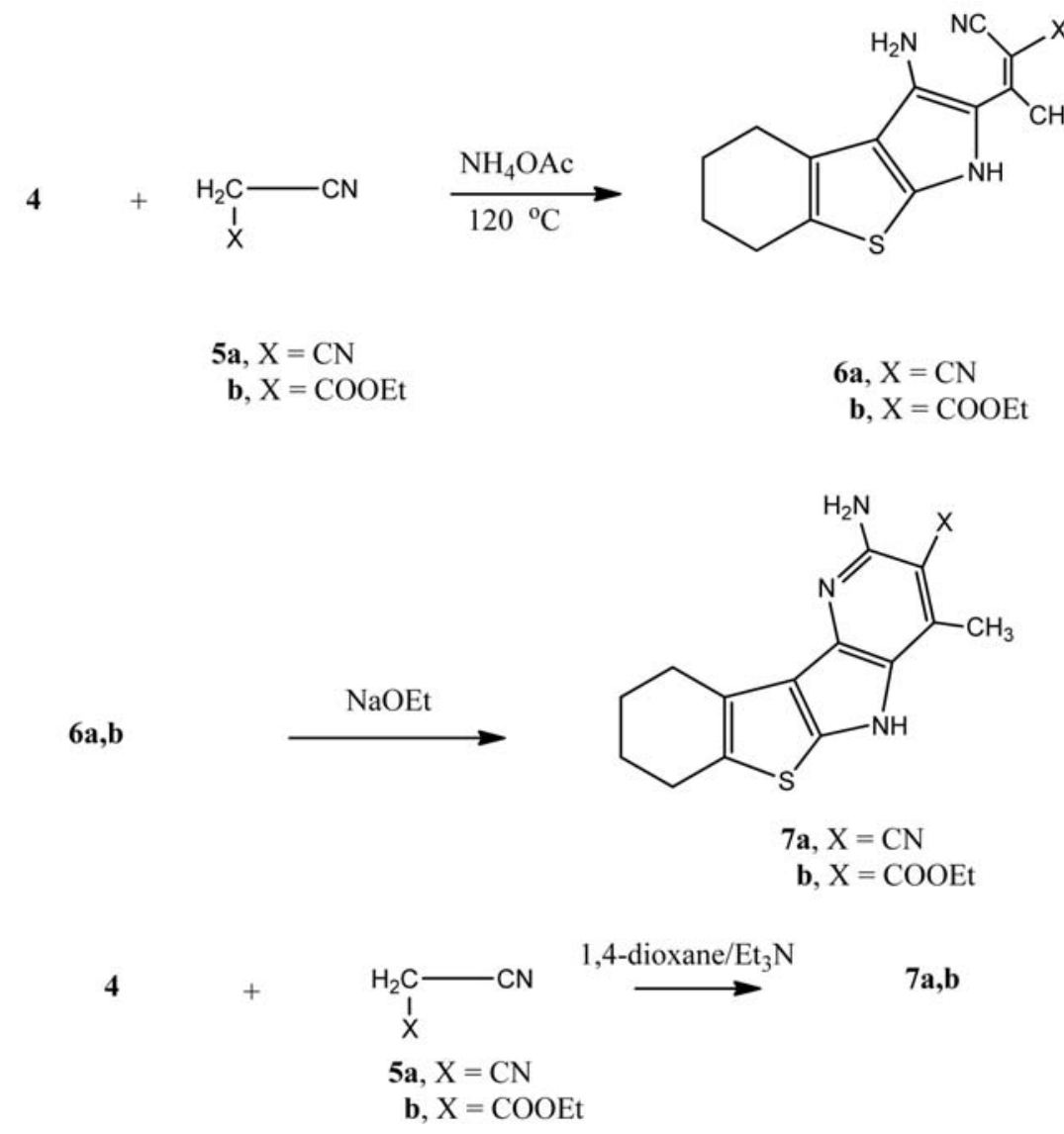

Shema 2. Synthesis of compounds $\mathbf{6 a , b}$ and $\mathbf{7 a , b}$. 
went ready cyclization when heated in sodium ethoxide solution in a boiling water bath to yield the 1-(3-amino4,5,6,7-tetrahydro- $1 H$-benzo[4,5] thieno[2,3-b]pyrrol-2yl)ethanone (4) (Scheme 1).

Compound 4 showed interesting reactivity towards different reagents, thus, it reacted with either malononitrile (5a) or ethyl cyanoacetate $(\mathbf{5 b})$ in the presence of ammonium acetate in an oil bath at $120{ }^{\circ} \mathrm{C}$ afforded the Knoevenagel condensated products $\mathbf{6 a}$ and $\mathbf{6 b}$, respectively. The latter products underwent ready cyclization in sodium ethoxide solution to give the annulated products 7a and 7b, respectively (Scheme 2). The structures of the latter products were established on the basis of the analytical and spectral data. Thus, the ${ }^{1} \mathrm{H}-\mathrm{NMR}$ spectrum of $\mathbf{7 a}$ showed the presence of $\delta 2.89$ ppm assigned to the $\mathrm{CH}_{3}$ group, a singlet at $\delta 4.89 \mathrm{ppm}$ indicating the $\mathrm{NH}_{2}$ group and a singlet at $\delta 8.33 \mathrm{ppm}$ confirming the presence of the $\mathrm{NH}$ group. Moreover, the ${ }^{13} \mathrm{C}$-NMR spectrum showed $\delta$
$19.8\left(\mathrm{CH}_{3}\right), 20.1,22.7,25.2$ and $34.6\left(4 \mathrm{CH}_{2}\right), 116.8$ (CN), 120.1, 122.6, 123.8, 124.2, 125.3, 127.2, 135.6, 142.3 (thiophene, pyrrole, pyridine $\mathrm{C}$ ) and $168.2(\mathrm{C}=\mathrm{N})$.

Compound 4 was studied to produce thiophene derivatives through the Gewald's reaction ${ }^{23-26}$ as many thiophenes were used as anticancer drugs. Thus, the reaction of compound 4 with either of malononitrile or ethyl cyanoacetate and elemental sulphur gave the thiophene derivatives $\mathbf{8 a}$ and $\mathbf{8 b}$, respectively. On the other hand, the one pot reaction of compound 4 with either malononitrile or ethyl cyanoacetate and any of benzaldehyde, 4-chlorobenzaldehyde or 4-methoxybenzaldehyde gave the pyran derivatives 10af, respectively. The ${ }^{1} \mathrm{H}-\mathrm{NMR}$ and ${ }^{13} \mathrm{C}-\mathrm{NMR}$ spectra 10a-f were consistent with their respective structures. Further confirmations for the structure of compounds 10a-f were obtained through their synthesis via another synthetic root. Thus, the reaction of compound $\mathbf{4}$ with the cinnamonitrile derivatives 11a-f in the presence of a catalytic amount of

$$
4+2 \text {, }
$$

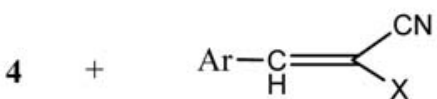

11a, $\mathrm{Ar}=\mathrm{C}_{6} \mathrm{H}_{5}, \mathrm{X}=\mathrm{CN}$

b, $\mathrm{Ar}=\mathrm{C}_{6} \mathrm{H}_{5}, \mathrm{X}=\mathrm{COOEt}$

c, $\mathrm{Ar}=4-\mathrm{Cl}-\mathrm{C}_{6} \mathrm{H}_{4}, \mathrm{X}=\mathrm{CN}$

d, $\mathrm{Ar}=4-\mathrm{Cl}-\mathrm{C}_{6} \mathrm{H}_{4}, \mathrm{X}=\mathrm{COOEt}$

e, $\mathrm{Ar}=4-\mathrm{OCH}_{3}, \mathrm{X}=\mathrm{CN}$

f, $\mathrm{Ar}=4-\mathrm{OCH}_{3}, \mathrm{R}=\mathrm{COOEt}$ 1,4-dioxane

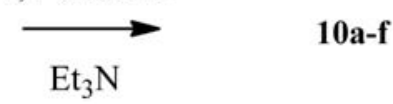

Shema 3. Synthesis of compounds $8 \mathbf{a}, \mathbf{b}$ and 10a-f. 
triethylamine gave the same products 10a-f, respectively (m.p., mixed m.p. and fingerprint IR) (Scheme 3).

Moreover, the reaction of either of compound 8a or $\mathbf{8 b}$ with ethyl cyanoacetate in refluxing dimethylformamide afforded the 2-amido derivatives $12 \mathbf{a}$ and $\mathbf{1 2 b}$, respectively. Formation of the latter products was explained on the condensation of ethyl cyanoacetate with the 2aminothiophene moiety not to the 3-aminopyrrol moiety on the basis of the ${ }^{1} \mathrm{H}$-NMR spectra of such products. Thus, the ${ }^{1} \mathrm{H}-\mathrm{NMR}$ spectrum of either 12a or 12b displayed the missing of the $\mathrm{NH}_{2}$ group that attached to thiophene ring which is expected to appear within the range $\delta$ 5.10-5.24 ppm while that of the 3-aminopyrrole moiety existing at $\delta 4.81-4.83 \mathrm{ppm}$. Similar acylation of the 2aminothiophene was reported before in literature. ${ }^{27}$ The high yield of compound 12a, encouraged us to make furt- her work. Thus, the reaction of 12a with either of the aryl diazonium salts 13a-d gave the aryl hydrazo derivatives 14a-d, respectively. Moreover, compounds 12a,b underwent ready cyclization in sodium ethoxide to produce the thieno[2,3- $b$ ]pyridine derivatives $\mathbf{1 5 a}$ and $\mathbf{1 5} \mathbf{b}$, respectively (Scheme 4).

\section{2. Anti-tumor Cell Activity}

\section{2. 1. Chemicals and Cell cultures}

Fetal bovine serum (FBS) and L-glutamine, were purchased from Gibco Invitrogen Co. (Scotland, UK). RPMI-1640 medium was purchased from Cambrex (New Jersey, USA). Dimethyl sulfoxide (DMSO), doxorubicin, CHS-828, penicillin, streptomycin and sulforhodamine B (SRB) were purchased from Sigma Chemical Co. (Saint

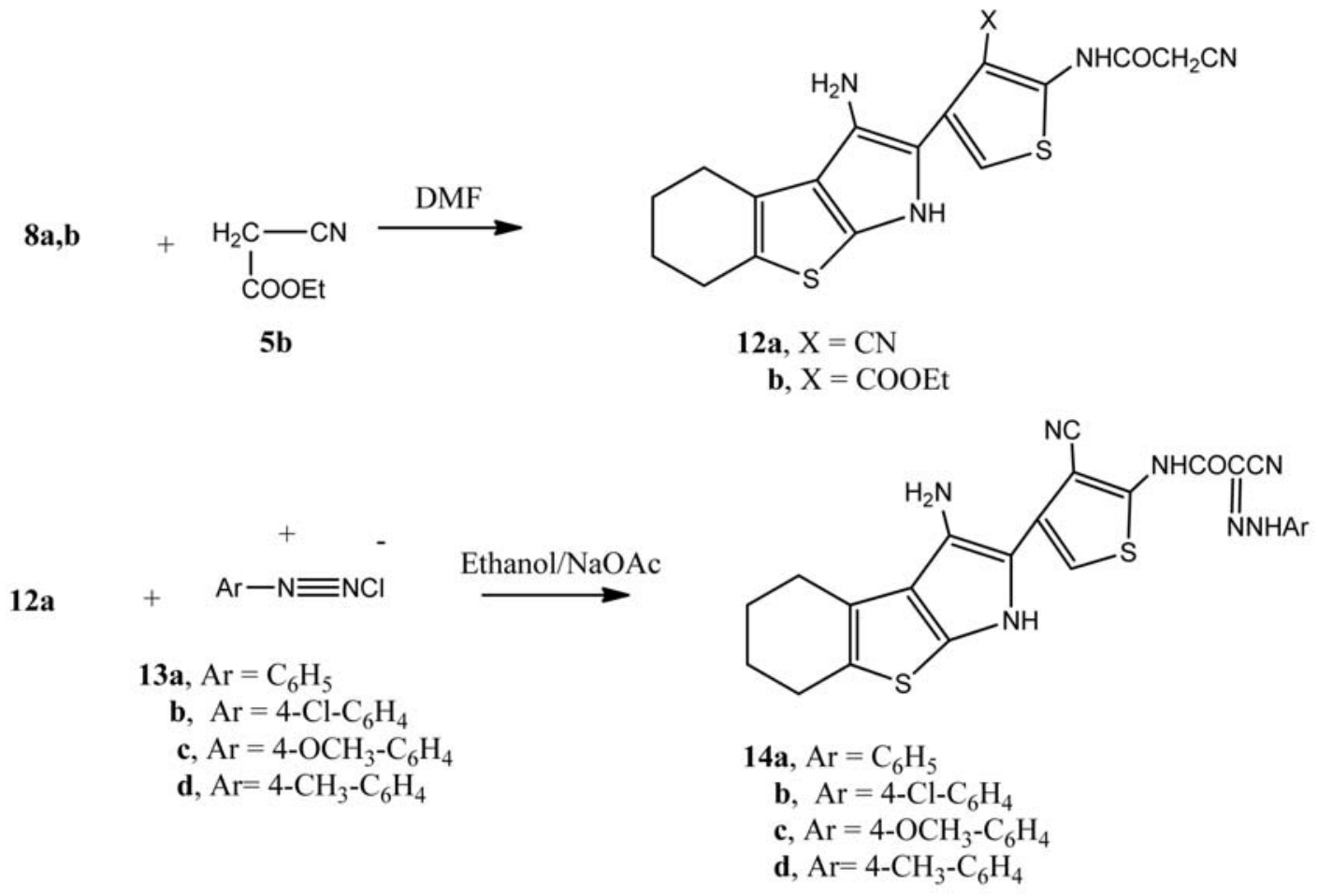<smiles>[R]c1c(C#N)c(O)nc2scc(-c3[nH]c4sc5c(c4c3N)CCCC5)c12</smiles>

15a, $\mathrm{R}=\mathrm{NH}_{2}$

b, $\mathrm{R}=\mathrm{OH}$

Shema 4. Synthesis of compounds 12a,b-15a,b. 
Louis, USA). The cell cultures was obtained from the European Collection of cell Cultures (ECACC, Salisbury, UK) and human gastric cancer (NUGC), human colon cancer (DLD-1), human liver cancer (HA22T and HEPG2), human breast cancer (MCF-7), nasopharyngeal carcinoma (HONE-1) and normal fibroblast cells (WI-38) were kindly provided by the National Cancer Institute (NCI, Cairo, Egypt). They grow as monolayer and routinely maintained in RPMI-1640 medium supplemented with $5 \%$ heat inactivated FBS, $2 \mathrm{mM}$ glutamine and antibiotics (penicillin $100 \mathrm{U} / \mathrm{mL}$, streptomycin $100 \mathrm{lg} / \mathrm{mL}$ ), at $37^{\circ} \mathrm{C}$ in a humidified atmosphere containing $5 \% \mathrm{CO}_{2}$. Exponentially growing cells were obtained by plating $1.5 \times 10^{5}$ cells/mL for the six human cancer cell lines including cells derived from $0.75 \times 10^{4}$ cells $/ \mathrm{mL}$ followed by $24 \mathrm{~h}$ of incubation. The effect of the vehicle solvent (DMSO) on the growth of these cell lines was evaluated in all the experiments by exposing untreated control cells to the maximum concentration $(0.5 \%)$ of DMSO used in each assay.

\section{2. 2. In vitro Cytotoxicity Assay}

The heterocyclic compounds, prepared in this study, were evaluated according to standard protocols ${ }^{28,29}$ for their in vitro cytotoxicity against the six human cancer cell lines including cells derived from human gastric cancer
(NUGC), human colon cancer (DLD-1), human liver cancer (HA22T and HEPG-2), human breast cancer (MCF-7), nasopharyngeal carcinoma (HONE-1) and a normal fibroblast cells (WI-38). All of $\mathrm{IC}_{50}$ values were listed in Table 1. Some heterocyclic compounds were observed with significant cytotoxicity against most of the cancer cell lines tested $\left(\mathrm{IC}_{50}=10-1000 \mathrm{nM}\right)$. Normal fibroblasts cells (WI-38) were affected to a much lesser extent $\left(\mathrm{IC}_{50}>10,000 \mathrm{nM}\right)$. The reference compound used was the CHS-828 which is the pyridyl cyanoguanidine anti-tumor agent. ${ }^{30}$ It is a new chemotherapeutic drug in addition it has low toxicity and lacks known patterns of multidrug resistance. ${ }^{31}$

\section{2. 3. Structure-activity Relationship}

From Table 1 it is clear that the thiophene moiety was found to be crucial for the cytotoxic effect of the cyclic compounds 3 -15a,b. Compounds $4,7 a, 7 b, 8 a, 8 b$, 10c, 10d, 10f, 12a, 12b, 14b and 15b exhibited optimal cytotoxic effect against cancer cell lines, with $\mathrm{IC}_{50}$ 's in the $\mathrm{nM}$ range. Comparing the cytotoxicity of the tetrahydrobenzothiophene $\mathbf{3}$ and the cyclized product $\mathbf{4}$, it is obvious that the cytotoxicity of compound $\mathbf{4}$ is higher than that of compound $\mathbf{3}$. The presence of the pyrrol ring through the tetrahydrobenzo[b]thiophene in compound $\mathbf{4}$ is responsible for its high potency. The condensation reac-

Table 1. Cytotoxicity of the newly synthesized products against a variety of cancer cell lines $\left[\mathrm{IC}_{50}{ }^{\mathrm{a}}(\mathrm{nM})\right]$

\begin{tabular}{|c|c|c|c|c|c|c|c|}
\hline \multirow{2}{*}{$\begin{array}{c}\text { Compound } \\
\text { No. }\end{array}$} & \multicolumn{7}{|c|}{ Cytotoxicity $\left(\mathrm{IC}_{50}\right.$ in $\left.\mathrm{nM}\right)$} \\
\hline & $\mathbf{U G C}^{\mathbf{b}}$ & DLD-1 $^{\mathrm{b}}$ & HA22T ${ }^{\mathrm{b}}$ & HEPG-2 $^{\mathrm{b}}$ & HONE-1 ${ }^{\text {b }}$ & MCF-7 ${ }^{\mathrm{b}}$ & WI-38 \\
\hline 3 & 2142 & 1222 & 1340 & 1028 & 1828 & 2246 & NA \\
\hline 4 & 86 & 45 & 313 & 128 & 212 & 310 & NA \\
\hline $6 \mathbf{a}$ & 2101 & 2380 & 3258 & 2266 & 2380 & 3330 & NA \\
\hline $6 \mathbf{b}$ & 1335 & 1140 & 1072 & 1154 & 1064 & 1258 & NA \\
\hline $7 a$ & 218 & 146 & 220 & 337 & 241 & 380 & NA \\
\hline $7 \mathbf{b}$ & 48 & 92 & 260 & 46 & 74 & 32 & NA \\
\hline $\mathbf{8 a}$ & 320 & 240 & 230 & 165 & 1281 & 265 & NA \\
\hline $8 b$ & 48 & 35 & 53 & 170 & 49 & 78 & NA \\
\hline $10 \mathrm{a}$ & 1220 & 1033 & 2250 & 1275 & 2126 & 2372 & NA \\
\hline 10b & 1165 & 1322 & 2350 & 2221 & 2152 & 1322 & NA \\
\hline $10 \mathrm{c}$ & 330 & 532 & 822 & 442 & 1529 & 1224 & NA \\
\hline 10d & 30 & 62 & 74 & 39 & 1330 & 88 & NA \\
\hline $10 \mathrm{e}$ & 1135 & 2160 & 2160 & 814 & 780 & 296 & NA \\
\hline $10 f$ & 149 & 2220 & 3210 & 550 & 2451 & 1286 & 120 \\
\hline $12 a$ & 69 & 74 & 190 & 448 & 2871 & 2690 & NA \\
\hline $12 b$ & 26 & 65 & 38 & 220 & 440 & 57 & NA \\
\hline $14 a$ & 1350 & 1160 & 2290 & 2120 & 1126 & 2230 & NA \\
\hline $14 b$ & 83 & 59 & 80 & 64 & 87 & 48 & 1330 \\
\hline $14 c$ & 1480 & 1156 & 1346 & 1226 & 1275 & 1240 & NA \\
\hline 14d & 1245 & 2160 & 2180 & 2220 & 1869 & 1765 & NA \\
\hline $15 a$ & 1845 & 1210 & 1218 & 1076 & 1270 & 436 & NA \\
\hline $15 b$ & 1220 & 2063 & 377 & 740 & 253 & 2210 & NA \\
\hline CHS- 828 & 25 & 2315 & 2067 & 1245 & 15 & 18 & NA \\
\hline
\end{tabular}

${ }^{\text {a }}$ Drug concentration required to inhibit tumor cell proliferation by $50 \%$ after continuous exposure of $48 \mathrm{~h}$.

${ }^{\mathrm{b}}$ NUGC, gastric cancer; DLD-1, colon cancer; HA22T, liver cancer; HEPG-2, liver cancer; HONE-1, nasopharyngeal carcinoma; MCF-7, breast cancer; WI-38, normal fibroblast cells. NA: Not Active. 
tion of compound $\mathbf{4}$ with either malononitrile or ethyl cyanoacetate to produce compounds $\mathbf{5 a}$ and $\mathbf{5 b}$, respectively showed a decrease of cytotoxicity. On the other hand, the cyclization of compounds $\mathbf{6 a}$ and $\mathbf{6 b}$ to the benzo[4', 5'] thieno [3',2':4,5]pyrrolo[3,2-b]pyridine derivatives $\mathbf{7 a}$ and $\mathbf{7 b}$ showed remarkable increase of the cytotoxicity. Moreover, it is clear that compound $\mathbf{7 b}$ showed more cytotoxicity than $\mathbf{7 a}$, this is attributed to the presence of the oxygen rich COOE-t group. The introduction of the second thiophene moiety to compound $\mathbf{4}$ that gives both of compounds $8 \mathbf{a}$ and $\mathbf{8 b}$ showed high potency-especially-in case of compounds $\mathbf{8 b}$ which was attributed due to the presence of the COOEt. Considering the pyran derivatives 10a-f, the cytotoxicity of compounds $10 \mathrm{c}$ and $10 \mathrm{~d}$ showed the highest values among the six compounds. However, compound 10c showed high cytotoxicity against the four cancer cell lines HUGC, DLD-1, HA22T and HEPG-2, but it is of great value to notice that compound 10d showed high cytotoxicity against five cancer cell lines and such cytotoxicity is higher than that of compound 10c. The high cytotoxicity of compound $\mathbf{1 0 d}$ is attributed to the presence of the $\mathrm{OH}$ and the $\mathrm{Cl}$ group as well.

The thiophene derivatives 12a and 12b showed high cytotoxicity similarl to that of compounds $\mathbf{8 a}, \mathbf{b}$. Moreover, compound $\mathbf{1 2 b}$ with the COOE-t showed high potency than that of compound 12a. The coupling of the diazonium salts 13a-d with compound 12a afforded the arylhydrazone derivatives 14a-d. Compound 14b with the $\mathrm{Cl}$ group showed the maximum cytotoxicity among the arylhydrazone derivatives 14a-d. Finally, considering the thieno[2,3- $b]$ pyridine derivatives 15a,b where the presence of the $\mathrm{OH}$ in compound $\mathbf{1 5 b}$ conserved an interesting cytotoxicity against the cancer cell lines HA22T, HEPG-2 and HONE-1 with the $\mathrm{IC}_{50}$ 's $377,740,253 \mathrm{nM}$, respectively. It is of great value to notice that compounds $\mathbf{7 b}, \mathbf{8 b}$ and $\mathbf{1 2 b}$ showed the maximum cytotoxicity among the tested compounds.

\section{2. 4. Anti-proliferative Cell Activity Against Cancer Cell Lines}

We used a panel of tumor cell lines to test the cytotoxicity of the new compounds, especially those showed high potency against the six cancer cell lines through Table 2. Importantly, this panel included the cell lines and their isogenic sub-lines with the determinants of drug resistance: murine leukemia L1210, T-lymphocyte cell lines Molt4/C8 and CEM, human leukemia R562 and its MDR subline K562/4 that over expressed P-glycoprotein, and the colon carcinoma HCT116. The above determinants alter the response of cells to many anticancer drugs including doxorubicin. Data on cytotoxic (anti-proliferative) activity are presented in Table 2 in which $\mathrm{IC}_{50}$ values represent the concentrations that inhibit cell proliferation by $50 \%$. It is clear from Table 2 that tested compounds $4,7 \mathbf{a}, 7 \mathbf{b}, \mathbf{8 a}, \mathbf{8 b}, \mathbf{1 0 c}$, 10d, 10f, 12a, 12b, 14b and 15b showed high potency against the cell lines. The benzo[4',5']thieno[ [3', 2':4,5]pyrrolo[3,2- $b]$ pyridine derivative $\mathbf{7 b}$ and the benzo[4,5]thieno[2,3-b]pyrrol-2-yl)-thiophene derivative $\mathbf{8 b}$ showed high potency against Molt4/C8 and CEM cell lines and their $\mathrm{IC}_{50}$ 's are higher than that of the reference doxorubicin. It is clear from Table 2 that the twelve tested compounds showed high $\mathrm{IC}_{50}$ against K562/4 cell line than doxorubicin.

\section{Experimental}

\section{1. General}

All melting points were determined on an electrothermal apparatus (Büchi 535, Switzerland) in an open capillary tube and are uncorrected. ${ }^{13} \mathrm{C}-\mathrm{NMR}$ and ${ }^{1} \mathrm{H}$-NMR spectra were recorded on Bruker DPX200 instrument in DMSO with TMS as internal standard for protons and solvent signals as internal standard for carbon spectra. Chemical shift values are mentioned in ä (ppm). Mass spectra

Table 2. Anti-proliferative activity $\left(\mathrm{IC}_{50}\right)$ of selected compounds against variety of cell lines

\begin{tabular}{|c|c|c|c|c|c|c|}
\hline Compound & & & Cytotoxi & $\mathrm{C}_{50}$ in $\left.\mathrm{nM}\right)$ & & \\
\hline No. & L1210 & Molt4/C8 & CEM & K562 & K562/4 & HCT116 \\
\hline 4 & $1.5 \pm 0.5$ & $1.1 \pm 0.03$ & $0.3 \pm 0.01$ & $0.4 \pm 0.08$ & $0.9 \pm 0.02$ & $0.8 \pm 0.05$ \\
\hline $7 \mathbf{a}$ & $0.4 \pm 0.1$ & $0.8 \pm 0.04$ & $2.0 \pm 0.4$ & $1.8 \pm 0.03$ & $0.9 \pm 0.06$ & $1.3 \pm 0.02$ \\
\hline $7 b$ & $0.3 \pm 0.08$ & $0.4 \pm 0.04$ & $0.9 \pm 0.05$ & $1.30 \pm 0.08$ & $1.1 \pm 0.07$ & $2.4 \pm 0.09$ \\
\hline $8 \mathbf{a}$ & $1.2 \pm 0.09$ & $0.8 \pm 0.02$ & $0.6 \pm 0.01$ & $0.2 \pm 0.01$ & $0.9 \pm 0.08$ & $1.4 \pm 0.2$ \\
\hline $8 b$ & $1.1 \pm 0.06$ & $0.02 \pm 0.002$ & $0.7 \pm 0.03$ & $0.9 \pm 0.06$ & $1.6 \pm 0.07$ & $0.8 \pm 0.02$ \\
\hline $10 \mathrm{c}$ & $0.8 \pm 0.05$ & $0.4 \pm 0.02$ & $1.3 \pm 0.05$ & $0.6 \pm 0.02$ & $0.02 \pm 0.01$ & $1.2 \pm 0.08$ \\
\hline 10d & $0.6 \pm 0.02$ & $1.5 \pm 0.07$ & $2.5 \pm 0.05$ & $1.7 \pm 0.02$ & $2.5 \pm 0.02$ & $2.8 \pm 0.07$ \\
\hline $10 f$ & $1.4 \pm 0.05$ & $0.8 \pm 0.03$ & $2.6 \pm 0.09$ & $0.02 \pm 0.01$ & $2.8 \pm 0.06$ & $0.4 \pm 0.08$ \\
\hline $12 a$ & $2.1 \pm 0.05$ & $0.6 \pm 0.02$ & $0.5 \pm 0.01$ & $0.3 \pm 0.01$ & $0.4 \pm 0.06$ & $2.4 \pm 0.07$ \\
\hline $12 b$ & $1.8 \pm 0.09$ & $0.9 \pm 0.04$ & $1.8 \pm 0.6$ & $0.7 \pm 0.06$ & $0.8 \pm 0.06$ & $0.9 \pm 0.08$ \\
\hline $14 b$ & $0.5 \pm 0.03$ & $0.3 \pm 0.05$ & $2.6 \pm 0.06$ & $0.5 \pm 0.07$ & $0.6 \pm 0.02$ & $0.1 \pm 0.01$ \\
\hline $15 b$ & $0.9 \pm 0.02$ & $0.3 \pm 0.01$ & $0.6 \pm 0.05$ & $2.1 \pm 0.07$ & $2.7 \pm 1.03$ & $0.3 \pm 0.04$ \\
\hline Dox. & $0.37 \pm 0.07$ & $0.20 \pm 0.02$ & $0.06 \pm 0.02$ & $0.14 \pm 0.03$ & $7.2 \pm 0.9$ & $1.4 \pm 0.1$ \\
\hline
\end{tabular}

Doxorubicin (Dox.) was used as the reference drug 
were recorded on EIMS (Shimadzu) and ESI-esquire 3000 Bruker Daltonics instrument. Elemental analyses were carried out by the Microanalytical Data Unit Ludwig-Maximilians-Universitat-Munchen, Germany. The progress of all reactions was monitored by TLC on $2 \times 5$ $\mathrm{cm}$ pre-coated silica gel 60 F254 plates of thickness of $0.25 \mathrm{~mm}$ (Merck).

\section{1. 1. Synthesis of 2-((2-Oxopropyl)amino)- 4,5,6,7-tetrahydrobenzo $[b]$ thiophene- 3-carbonitrile (3)}

To a solution of compound $\mathbf{1}(1.78 \mathrm{~g}, 0.01 \mathrm{~mol})$ in 1,4-dioxane $(40 \mathrm{~mL})$ containing sodium carbonate $(1.00 \mathrm{~g})$ $\alpha$-chloroacetone $(0.94 \mathrm{~g}, 0.01 \mathrm{~mol})$ was added. The reaction mixture was heated under reflux for $2 \mathrm{~h}$ then poured onto ice/water and the formed solid product was collected by filtration and crystallized from ethanol.

White crystals; yield: $2.01 \mathrm{~g}(86 \%)$; mp: 182-183 ${ }^{\circ} \mathrm{C}$; IR $\left(\mathrm{KBr}, \mathrm{cm}^{-1}\right)$ : 3465-3328 (NH), $2220(\mathrm{CN})$, $1705(\mathrm{C}=\mathrm{O}), 1615(\mathrm{C}=\mathrm{C}) ;{ }^{1} \mathrm{H}-\mathrm{NMR}$ (dimethyl sulfoxide (DMSO)- $d_{6}$ ) $\delta: 1.80-1.85\left(\mathrm{~m}, 4 \mathrm{H}, 2 \mathrm{CH}_{2}\right), 2.22-2.26(\mathrm{~m}$, $\left.4 \mathrm{H}, 2 \mathrm{CH}_{2}\right), 2.88\left(\mathrm{~s}, 3 \mathrm{H}, \mathrm{CH}_{3}\right), 5.20\left(\mathrm{~s}, 2 \mathrm{H}, \mathrm{CH}_{2}\right), 8.30(\mathrm{~s}$, $1 \mathrm{H}, \mathrm{NH}, \mathrm{D}_{2} \mathrm{O}$ exchangeable); ${ }^{13} \mathrm{C}-\mathrm{NMR}$ (DMSO-d $d_{6} \delta$ : 19.6, 20.3, 22.0, 25.7, 34.6, 55.6, 116.8, 124.1, 124.9, 128.7, 139.5, 164.8; MS electron impact (EI): $\mathrm{m} / \mathrm{z}(\%)$ $234\left(\mathrm{M}^{+}\right)$. Anal. Calcd for $\mathrm{C}_{12} \mathrm{H}_{14} \mathrm{~N}_{2} \mathrm{OS}: \mathrm{C}, 61.51 ; \mathrm{H}$, 6.02; N, 11.96; S, 13.68. Found: C, 61.82; H, 6.22; N, 11.77; S, 13.73 .

Synthesis of 1-(3-Amino-4,5,6,7-tetrahydro- $1 H$-benzo[4,5] thieno[2,3-b]pyrrol-2-yl)ethanone (4)

A suspension of compound $3(2.34 \mathrm{~g}, 0.01 \mathrm{~mol})$ in sodium ethoxide $(0.02 \mathrm{~mol})$ [prepared by dissolving metallic sodium $(0.46 \mathrm{~g}, 0.02 \mathrm{~g})$ in absolute ethanol $(20 \mathrm{~mL}]$ was heated in a boiling water bath for $6 \mathrm{~h}$ then poured onto ice/water containing few drops of hydrochloric acid. The formed solid product was collected by filtration and crystallized from 1,4-dioxane.

White crystals; yield: $1.80 \mathrm{~g}(77 \%)$; $\mathrm{mp}:>300{ }^{\circ} \mathrm{C}$; IR $\left(\mathrm{KBr}, \mathrm{cm}^{-1}\right)$ : 3479-3348 $\left(\mathrm{NH}, \mathrm{NH}_{2}\right), 1715(\mathrm{C}=\mathrm{O})$, $1618(\mathrm{C}=\mathrm{C}) ;{ }^{1} \mathrm{H}-\mathrm{NMR}$ (DMSO- $\left.d_{6}\right) \delta: 1.78-1.83(\mathrm{~m}, 4 \mathrm{H}$, $\left.2 \mathrm{CH}_{2}\right), 2.20-2.27\left(\mathrm{~m}, 4 \mathrm{H}, 2 \mathrm{CH}_{2}\right), 2.91\left(\mathrm{~s}, 3 \mathrm{H}, \mathrm{CH}_{3}\right), 4.83$ (s, $2 \mathrm{H}, \mathrm{NH}_{2}, \mathrm{D}_{2} \mathrm{O}$ exchangeable), $8.27\left(\mathrm{~s}, 1 \mathrm{H}, \mathrm{NH}, \mathrm{D}_{2} \mathrm{O}\right.$ exchangeable); ${ }^{13} \mathrm{C}-\mathrm{NMR}$ (DMSO- $\left.d_{6}\right) \delta: 19.8,20.2,22.0$, 25.6, 34.8, 124.0, 124.9, 128.5, 139.6, 165.6; MS (EI): $\mathrm{m} / z(\%) 234\left(\mathrm{M}^{+}\right)$. Anal. Calcd for $\mathrm{C}_{12} \mathrm{H}_{14} \mathrm{~N}_{2} \mathrm{OS}$ : C, 61.51; H, 6.02; N, 11.96; S, 13.68. Found: C, 61.68; H, 5.89; N, $12.20 ; \mathrm{S}, 13.83$.

\section{1. 2. General Procedure for the Synthesis of Thieno[2,3-b]pyrrol Derivatives 6a and $6 \mathrm{~b}$}

To the dry solid of compound 4 (2.34 g, $0.01 \mathrm{~mol})$ either malononitrile $(0.66 \mathrm{~g}, 0.01 \mathrm{~mol})$ or ethyl cyanoacetate $(1.13 \mathrm{~g}, 0.01 \mathrm{~mol})$ was added followed by ammonium acetate $(0.50 \mathrm{~g}, 0.01 \mathrm{~mol})$. The whole reaction mixture was heated in an oil bath at $120^{\circ} \mathrm{C}$ for $1 \mathrm{~h}$ then left to cool. The solidified product was boiled with ethanol then left to cool. The formed solid product was collected by filtration and crystallized from acetic acid.

2-(1-(3-Amino-4,5,6,7-tetrahydro-1H-benzo[4,5]thieno[2,3-b]pyrrol-2-yl)ethylidene)-malononitrile (6a)

Yellow crystals; yield: $1.92 \mathrm{~g}$ (68\%); mp: 167-168 ${ }^{\circ} \mathrm{C}$; IR $\left(\mathrm{KBr}, \mathrm{cm}^{-1}\right)$ : 3488-3334 $\left(\mathrm{NH}, \mathrm{NH}_{2}\right), 3054(\mathrm{CH}$ aromatic), 2227, $2222(2 \mathrm{CN}), 1620(\mathrm{C}=\mathrm{C}) ;{ }^{1} \mathrm{H}-\mathrm{NMR}$ (DMSO- $d_{6}$ ) $\delta: 1.79-1.86\left(\mathrm{~m}, 4 \mathrm{H}, 2 \mathrm{CH}_{2}\right),\left(\mathrm{m}, 4 \mathrm{H}, 2 \mathrm{CH}_{2}\right)$, 2.69 (s, $\left.3 \mathrm{H}, \mathrm{CH}_{3}\right), 4.86$ (s, $2 \mathrm{H}, \mathrm{NH}_{2}, \mathrm{D}_{2} \mathrm{O}$ exchangeable), $8.29\left(\mathrm{~s}, \quad 1 \mathrm{H}, \quad \mathrm{NH}, \quad \mathrm{D}_{2} \mathrm{O}\right.$ exchangeable); ${ }^{13} \mathrm{C}-\mathrm{NMR}$ (DMSO- $d_{6}$ ) $\delta: 19.4,20.3,22.2,25.6,34.5,116.3,116.9$, $122.3,123.8,124.0,124.9,127.2,135.2 ; \mathrm{MS}(\mathrm{EI}): \mathrm{m} / \mathrm{z}$ (\%) $282\left(\mathrm{M}^{+}\right)$. Anal. Calcd for $\mathrm{C}_{15} \mathrm{H}_{14} \mathrm{~N}_{4} \mathrm{~S}: \mathrm{C}, 63.80 ; \mathrm{H}$, 5.00; N, 19.84; S, 11.36. Found: C, 63.72; H, 4.93; N, $20.05 ;$ S, 11.59 .

Ethyl 3-(3-amino-4,5,6,7-tetrahydro-1H-benzo[4,5]thieno[2,3-b]pyrrol-2-yl)-2-cyanobut-2-enoate (6b)

Yellow crystals; yield: $2.46 \mathrm{~g}(75 \%)$; $\mathrm{mp}$ : 121-122 ${ }^{\circ} \mathrm{C}$; IR $\left(\mathrm{KBr}, \mathrm{cm}^{-1}\right): 3473-3330\left(\mathrm{NH}, \mathrm{NH}_{2}\right), 3054$ (CH aromatic), $2222(\mathrm{CN}), 1640(\mathrm{C}=\mathrm{C}) ;{ }^{1} \mathrm{H}-\mathrm{NMR}$ $\left(\right.$ DMSO- $\left.d_{6}\right) \delta: 1.13\left(\mathrm{t}, 3 \mathrm{H}, J=7.26 \mathrm{~Hz}, \mathrm{CH}_{3}\right), 1.80-1.86$ $\left(\mathrm{m}, 4 \mathrm{H}, 2 \mathrm{CH}_{2}\right), 2.22-2.27\left(\mathrm{~m}, 4 \mathrm{H}, 2 \mathrm{CH}_{2}\right), 2.66(\mathrm{~s}, 3 \mathrm{H}$, $\left.\mathrm{CH}_{3}\right), 4.22\left(\mathrm{q}, 2 \mathrm{H}, J=7.26 \mathrm{~Hz}, \mathrm{CH}_{2}\right), 4.88\left(\mathrm{~s}, 2 \mathrm{H}, \mathrm{NH}_{2}\right.$, $\mathrm{D}_{2} \mathrm{O}$ exchangeable), 8.27 (s, 1H, NH, $\mathrm{D}_{2} \mathrm{O}$ exchangeable); ${ }^{13} \mathrm{C}$-NMR (DMSO- $d_{6}$ ) $\delta$ : 16.3, 19.6, 20.2, 22.5, 25.6, 34.8, 116.6, 122.0, 123.5, 124.6, 124.7, 127.2, 134.8, 166.1; MS (EI): $\mathrm{m} / \mathrm{z}(\%) 329\left(\mathrm{M}^{+}\right)$. Anal. Calcd for $\mathrm{C}_{17} \mathrm{H}_{19} \mathrm{~N}_{3} \mathrm{O}_{2} \mathrm{~S}: \mathrm{C}, 61.98 ; \mathrm{H}, 5.81 ; \mathrm{N}, 12.76 ; \mathrm{S}, 9.73$. Found: C, 62.08; H, 6.07; N, 12.59; S, 9.88.

\section{1. 3. General Procedure for the Synthesis of the Benzo[4',5']thieno[3',2':4,5]-pyrrolo $[3,2-b]$ pyridine Derivatives $7 \mathrm{a}$ and $7 \mathrm{~b}$}

Method (A): A suspension of either compound $\mathbf{6 a}$ $(2.28 \mathrm{~g}, 0.01 \mathrm{~mol})$ or $\mathbf{6 b}(3.29 \mathrm{~g}, 0.01 \mathrm{~mol})$ in sodium ethoxide $(0.02 \mathrm{~mol})$ [prepared by dissolving metallic sodium $(0.46 \mathrm{~g}, 0.02 \mathrm{~mol})$ in absolute ethanol $(20 \mathrm{~mL})$ was heated in a boiling water bath for $8 \mathrm{~h}$ then poured onto ice/water containing few drops of hydrochloric acid. The formed solid product was collected by filtration and crystallized from acetic acid.

Method (B): To a solution of compound $4(2.34 \mathrm{~g}$, $0.01 \mathrm{~mol})$ in 1,4 -dioxane $(40 \mathrm{~mL})$ containing triethylamine $(0.50 \mathrm{~mL})$ either malononitrile $(0.66 \mathrm{~g}, 0.01 \mathrm{~mol})$ or ethyl cyanoacetate $(1.13 \mathrm{~g}, 0.01 \mathrm{~mol})$ was added. The whole reaction mixture, in each case, was heated under reflux for $4 \mathrm{~h}$ then poured onto ice/water containing few drops of hydrochloric acid. The formed solid product was collected by filtration and crystallized from acetic acid. 
2-Amino-4-methyl-7,8,9,10-tetrahydro-5 $H$-benzo $\left[4^{\prime}, 5^{\prime}\right]$ thieno[3',2':4,5]pyrrolo[3,2-b]pyridine-3-carbonitrile (7a)

Yellow crystals; yield: $2.27 \mathrm{~g}$ (80\%); mp: 232-233 ${ }^{\circ} \mathrm{C}$; IR $\left(\mathrm{KBr}, \mathrm{cm}^{-1}\right)$ : 3474-3314 $\left(\mathrm{NH}, \mathrm{NH}_{2}\right), 3056(\mathrm{CH}$ aromatic), $2220(\mathrm{CN}), 1626(\mathrm{C}=\mathrm{C}) ;{ }^{1} \mathrm{H}-\mathrm{NMR}$ (DMSO- $d_{6}$ ) $\delta: 1.76-1.84\left(\mathrm{~m}, 4 \mathrm{H}, 2 \mathrm{CH}_{2}\right), 2.21-2.26\left(\mathrm{~m}, 4 \mathrm{H}, 2 \mathrm{CH}_{2}\right)$, 2.89 (s, $\left.3 \mathrm{H}, \mathrm{CH}_{3}\right), 4.89$ (s, 2H, $\mathrm{NH}_{2}, \mathrm{D}_{2} \mathrm{O}$ exchangeable), $8.33\left(\mathrm{~s}, \quad 1 \mathrm{H}, \quad \mathrm{NH}, \quad \mathrm{D}_{2} \mathrm{O}\right.$ exchangeable); ${ }^{13} \mathrm{C}-\mathrm{NMR}$ (DMSO- $d_{6}$ ) $\delta: 19.8,20.1,22.7,25.2,34.6,116.8,120.1$, 122.6, 123.8, 124.2, 125.3, 127.2, 135.6, 142.3, 168.2; MS (EI): $\mathrm{m} / z(\%) 282\left(\mathrm{M}^{+}\right)$. Anal. Calcd for $\mathrm{C}_{15} \mathrm{H}_{14} \mathrm{~N}_{4} \mathrm{~S}$ : C, 63.80; H, 5.00; N, 19.84; S, 11.36. Found: C, 63.66; H, $4.83 ; \mathrm{N}, 20.25 ; \mathrm{S}, 11.37$.

Ethyl 2-amino-4-methyl-7,8,9,10-tetrahydro-5 $H$-benzo $\left[4^{\prime}, 5^{\prime}\right]$ thieno $\left[3^{\prime}, 2^{\prime}: 4,5\right]$ pyrrolo [3,2-b]pyridine-3-carboxylate (7b)

Yellow crystals; yield: $2.24 \mathrm{~g}$ (68\%), mp: 195-196 ${ }^{\circ} \mathrm{C}$; IR $\left(\mathrm{KBr}, \mathrm{cm}^{-1}\right)$ : 3466-3327 $\left(\mathrm{NH}, \mathrm{NH}_{2}\right), 3056(\mathrm{CH}$ aromatic), $1640(\mathrm{C}=\mathrm{C}) ;{ }^{1} \mathrm{H}-\mathrm{NMR}$ (DMSO- $\left.d_{6}\right) \delta: 1.14(\mathrm{t}$, $\left.3 \mathrm{H}, J=7.07 \mathrm{~Hz}, \mathrm{CH}_{3}\right), 1.82-1.86\left(\mathrm{~m}, 4 \mathrm{H}, 2 \mathrm{CH}_{2}\right)$, 2.20-2.27 (m, 4H, 2 $\left.\mathrm{CH}_{2}\right), 2.88\left(\mathrm{~s}, 3 \mathrm{H}, \mathrm{CH}_{3}\right), 4.24(\mathrm{q}, 2 \mathrm{H}$, $\left.J=7.07 \mathrm{~Hz}, \mathrm{CH}_{2}\right), 4.84\left(\mathrm{~s}, 2 \mathrm{H}, \mathrm{NH}_{2}, \mathrm{D}_{2} \mathrm{O}\right.$ exchangeable), 8.32 (s, $1 \mathrm{H}, \mathrm{NH}, \mathrm{D}_{2} \mathrm{O}$ exchangeable); ${ }^{13} \mathrm{C}-\mathrm{NMR}$ (DMSO- $d_{6}$ ) $\delta: 16.2,19.8,20.3,22.5,25.6,34.5,55.6,120.3,122.4$, 123.8, 124.6, 124.7, 127.6, 133.9, 143.2, 164.4, 168.9; MS (EI): $\mathrm{m} / z(\%) 329\left(\mathrm{M}^{+}\right)$. Anal. Calcd for $\mathrm{C}_{17} \mathrm{H}_{19} \mathrm{~N}_{3} \mathrm{O}_{2} \mathrm{~S}$ : C, 61.98; H, 5.81; N, 12.76; S, 9.73. Found: C, 61.68; H, 5.94; N, 12.63; S, 9.90.

\section{1. 4. General Procedure for the Synthesis of $[4,5]$ thieno $[2,3-b]$ pyrrol-2-yl)thiophene Derivatives 8a and $8 \mathrm{~b}$}

To a solution of compound $4(2.34 \mathrm{~g}, 0.01 \mathrm{~mol})$ in 1,4-dioxane $(40 \mathrm{~mL})$ containing triethylamine $(0.50 \mathrm{~mL})$ and elemental sulfur $(0.32 \mathrm{~g}, 0.01 \mathrm{~mol})$ either malononitrile $(0.66 \mathrm{~g}, 0.01 \mathrm{~mol})$ or ethyl cyanoacetate $(1.13 \mathrm{~g}, 0.01$ mol) was added. The reaction mixture, in each case was heated under reflux for $2 \mathrm{~h}$ then was left to cool and the formed solid product, in each case, was collected by filtration and crystallized from ethanol.

2-Amino-4-(3-amino-4,5,6,7-tetrahydro- $1 H$-benzo $[4,5]$ thieno $[2,3-b]$ pyrrol-2-yl)thiophene-3-carbonitrile (8a)

Orange crystals; yield: $2.42 \mathrm{~g}$ (77\%), mp: 141-142 ${ }^{\circ} \mathrm{C}$; IR $\left(\mathrm{KBr}, \mathrm{cm}^{-1}\right)$ : 3462-3354 (NH, $\left.\mathrm{NH}_{2}\right), 3053(\mathrm{CH}$ aromatic), $2221(\mathrm{CN}), 1628(\mathrm{C}=\mathrm{C}) ;{ }^{1} \mathrm{H}-\mathrm{NMR}$ (DMSO- $\left.d_{6}\right)$ $\delta: 1.78-1.84\left(\mathrm{~m}, 4 \mathrm{H}, 2 \mathrm{CH}_{2}\right), 2.23-2.28\left(\mathrm{~m}, 4 \mathrm{H}, 2 \mathrm{CH}_{2}\right)$, 4.80, $5.25\left(2 \mathrm{~s}, 4 \mathrm{H}, 2 \mathrm{NH}_{2}, \mathrm{D}_{2} \mathrm{O}\right.$ exchangeable), $6.11(\mathrm{~s}, 1 \mathrm{H}$, thiophene $\mathrm{H}-5), 8.26$ ( $, 1 \mathrm{H}, \mathrm{NH}, \mathrm{D}_{2} \mathrm{O}$ exchangeable); ${ }^{13} \mathrm{C}-\mathrm{NMR}\left(\mathrm{DMSO}-d_{6}\right) \delta: 20.4,22.9,25.0,34.6,116.6$, 120.3, 123.1, 123.8, 124.2, 125.3, 127.2, 139.3, 140.6, 142.3; MS (EI): $m / z(\%) 314\left(\mathrm{M}^{+}\right)$. Anal. Calcd for
$\mathrm{C}_{15} \mathrm{H}_{14} \mathrm{~N}_{4} \mathrm{~S}_{2}: \mathrm{C}, 57.30 ; \mathrm{H}, 4.49 ; \mathrm{N}, 17.82 ; \mathrm{S}, 20.40$. Found: C, 57.44; H, 4.39; N, 18.04; S, 20.28.

Ethyl 2-amino-4-(3-amino-4,5,6,7-tetrahydro-1H-benzo[4,5] thieno[2,3-b]pyrrol-2-yl)-thiophene-3-carboxylate (8b)

Orange crystals; yield: $2.60 \mathrm{~g}$ (74\%), mp: 131-132 ${ }^{\circ} \mathrm{C}$. IR $\left(\mathrm{KBr}, \mathrm{cm}^{-1}\right)$ : 3479-3331 $\left(\mathrm{NH}_{2}\right), 3053(\mathrm{CH}$ aromatic), $1690(\mathrm{C}=\mathrm{O}), 1632(\mathrm{C}=\mathrm{C}) ;{ }^{1} \mathrm{H}-\mathrm{NMR}\left(\mathrm{DMSO}-d_{6}\right) \delta$ : 1.13 (t, $3 \mathrm{H}, J=6.83 \mathrm{~Hz}, \mathrm{CH}_{3}$ ), 1.81-1.87 (m, $4 \mathrm{H}, 2 \mathrm{CH}_{2}$ ), $2.22-2.25\left(\mathrm{~m}, 4 \mathrm{H}, 2 \mathrm{CH}_{2}\right), 4.23\left(\mathrm{q}, 2 \mathrm{H}, J=6.83 \mathrm{~Hz}, \mathrm{CH}_{2}\right.$ ), 4.81, $5.03\left(2 \mathrm{~s}, 4 \mathrm{H}, 2 \mathrm{NH}_{2}, \mathrm{D}_{2} \mathrm{O}\right.$ exchangeable $), 6.13(\mathrm{~s}, 1 \mathrm{H}$, thiophene $\mathrm{H}-5), 8.30\left(\mathrm{~s}, 1 \mathrm{H}, \mathrm{D}_{2} \mathrm{O}\right.$ exchangeable); ${ }^{13} \mathrm{C}-\mathrm{NMR}$ (DMSO- $d_{6}$ ) $\delta$ : 16.0, 20.0, 22.7, 25.6, 34.5, 55.6, 120.8, 122.7, 123.8, 124.6, 124.9, 127.6, 133.9, 143.5, 164.2; MS (EI): $\mathrm{m} / z$ (\%) $361\left(\mathrm{M}^{+}\right)$. Anal. Calcd for $\mathrm{C}_{17} \mathrm{H}_{19} \mathrm{~N}_{3} \mathrm{O}_{2} \mathrm{~S}_{2}$ : C, 56.48; H, 5.30; N, 11.62; S, 17.74. Found: C, 56.71; H, 5.55; N, 11.42; S, 17.49.

\section{1. 5. General Procedure for the Synthesis of Pyran Derivatives 10a-f}

Method (A): General procedure: To a solution of compound $4(2.34 \mathrm{~g}, 0.01 \mathrm{~mol})$ in 1,4-dioxane $(40 \mathrm{~mL})$ containing triethylamine $(0.5 \mathrm{~mL})$, either of malononitrile $(0.66 \mathrm{~g}, 0.01 \mathrm{~mol})$ or ethyl cyanoacetate $(1.13 \mathrm{~g}, 0.01 \mathrm{~mol})$ and either of benzaldehyde $(1.06 \mathrm{~g}, 0.1 \mathrm{~mol})$, 4-chlorobenzaldehyde $(1.40 \mathrm{~g}, 0.01 \mathrm{~mol})$ or 4-methoxybenzaldehyde (1.36 g, $0.01 \mathrm{~mol})$ were added. The reaction mixture was heated under reflux for $1 \mathrm{~h}$ and the formed solid product produced from the hot solution was collected by filtration and crystallized from ethanol. Thin layer chromatography revealed just a single spot which proved the presence of a single product.

Method (B): To a solution of compound 4 (2.34 g, $0.01 \mathrm{~mol})$ in 1,4-dioxane $(40 \mathrm{~mL})$ containing triethylamine $(0.5 \mathrm{~mL})$, either of the cinnamonitrile derivatives 11a-f ( $0.01 \mathrm{~mol})$ were added. The reaction mixture was heated under reflux for $2 \mathrm{~h}$ and the formed solid product produced from the hot solution was collected by filtration and crystallized from ethanol. Thin layer chromatography revealed just a single spot which proved the presence of a single product.

2-Amino-6-(3-amino-4,5,6,7-tetrahydro- $1 H$-benzo $[4,5]$ thieno[2,3-b]pyrrol-2-yl)-4- phenyl-4H-pyran-3carbonitrile (10a)

Pale yellow crystals; yield: $3.10 \mathrm{~g}(80 \%)$; mp: 167-168 ${ }^{\circ}$; IR $\left(\mathrm{KBr}, \mathrm{cm}^{-1}\right)$ : 3489-3321 $\left(\mathrm{NH}, \mathrm{NH}_{2}\right)$, $3056\left(\mathrm{CH}\right.$ aromatic), $2220(\mathrm{CN}), 1630(\mathrm{C}=\mathrm{C}) ;{ }^{1} \mathrm{H}-\mathrm{NMR}$ (DMSO- $d_{6}$ ) $\delta: 1.76-1.85\left(\mathrm{~m}, 4 \mathrm{H}, 2 \mathrm{CH}_{2}\right), 2.21-2.27(\mathrm{~m}$, $\left.4 \mathrm{H}, 2 \mathrm{CH}_{2}\right), 4.83,5.41\left(2 \mathrm{~s}, 4 \mathrm{H}, 2 \mathrm{NH}_{2}, \mathrm{D}_{2} \mathrm{O}\right.$ exchangeable), 5.66-5.90 (2d, 2H, pyran H-4, H-5), 7.28-7.38 (m, 5H, $\left.\mathrm{C}_{6} \mathrm{H}_{5}\right), 8.24$ (s, $1 \mathrm{H}, \mathrm{NH}, \mathrm{D}_{2} \mathrm{O}$ exchangeable); ${ }^{13} \mathrm{C}-\mathrm{NMR}$ (DMSO- $d_{6}$ ) $\delta: 20.6,22.9,25.3,34.8,39.3,116.9,120.6$, $122.8,123.8,123.9,125.3,126.9,127.2,129.4,130.8$, 
139.3, 140.6, 141.8, 142.3; MS (EI): $\mathrm{m} / \mathrm{z}(\%) 388\left(\mathrm{M}^{+}\right)$. Anal. Calcd for $\mathrm{C}_{22} \mathrm{H}_{20} \mathrm{~N}_{4} \mathrm{OS}: \mathrm{C}, 68.02 ; \mathrm{H}, 5.19 ; \mathrm{N}, 14.42$; S, 8.25. Found: C, 67.93; H, 5.32; N, 14.60; S, 8.44.

6-(3-Amino-4,5,6,7-tetrahydro-1H-benzo[4,5] thieno [2,3-b]pyrrol-2-yl)-2-hydroxy-4-phenyl-4H-pyran-3carbonitrile (10b)

Pale yellow crystals; yield: $2.57 \mathrm{~g}$ (66\%), mp: 264-265 ${ }^{\circ} \mathrm{C}$; IR (KBr, cm $\left.{ }^{-1}\right): 3520-3341\left(\mathrm{NH}, \mathrm{NH}_{2}, \mathrm{OH}\right)$, 3055 (CH aromatic), $2222(\mathrm{CN}), 1632(\mathrm{C}=\mathrm{C})$; ${ }^{1} \mathrm{H}-\mathrm{NMR}$ $\left(\mathrm{DMSO}-d_{6}\right) \delta$ : 1.77-1.86 (m, 4H, 2CH$), 2.20-2.27(\mathrm{~m}$, $\left.4 \mathrm{H}, 2 \mathrm{CH}_{2}\right), 4.86\left(\mathrm{~s}, 2 \mathrm{H}, \mathrm{NH}_{2}, \mathrm{D}_{2} \mathrm{O}\right.$ exchangeable $)$, 5.68-5.87 (2d, 2H, pyran H-4, H-5), 7.30-7.41 (m, 5H, $\left.\mathrm{C}_{6} \mathrm{H}_{5}\right), 8.22$ (s, 1H, NH, $\mathrm{D}_{2} \mathrm{O}$ exchangeable), $10.30(\mathrm{~s}, 1 \mathrm{H}$, $\mathrm{OH}, \mathrm{D}_{2} \mathrm{O}$ exchangeable); ${ }^{13} \mathrm{C}-\mathrm{NMR}$ (DMSO- $\left.d_{6}\right) \delta: 20.4$, 22.7, 25.4, 34.8, 39.9, 116.7, 120.8, 122.8, 123.3, 123.9, 125.7, 126.9, 127.0, 130.4, 133.6, 139.3, 140.8, 142.0, 142.7; MS (EI): $\mathrm{m} / z$ (\%) $389\left(\mathrm{M}^{+}\right)$. Anal. Calcd for $\mathrm{C}_{22} \mathrm{H}_{19} \mathrm{~N}_{3} \mathrm{O}_{2} \mathrm{~S}$ : C, 67.84; H, 4.92; N, 10.79; S, 8.23. Found: C, 67.60; H, 4.69; N, 10.99; S, 8.40.

2-Amino-6-(3-amino-4,5,6,7-tetrahydro-1H-benzo $[4,5]$ thieno[2,3-b]pyrrol-2-yl)-4-(4-chlorophenyl-4Hpyran-3-carbonitrile (10c)

Pale yellow crystals; yield: $2.87 \mathrm{~g}$ (68\%); mp: 274-275 ${ }^{\circ} \mathrm{C}$; IR $\left(\mathrm{KBr}, \mathrm{cm}^{-1}\right)$ : 3474-3330 (NH, $\left.\mathrm{NH}_{2}\right)$, 3055 (CH aromatic), $2220(\mathrm{CN}), 1633(\mathrm{C}=\mathrm{C})$; ${ }^{1} \mathrm{H}-\mathrm{NMR}$ $\left(\mathrm{DMSO}-d_{6}\right) \delta$ : $1.78-1.85\left(\mathrm{~m}, 4 \mathrm{H}, 2 \mathrm{CH}_{2}\right), 2.18-2.25(\mathrm{~m}$, $\left.4 \mathrm{H}, 2 \mathrm{CH}_{2}\right), 4.86,5.40\left(2 \mathrm{~s}, 4 \mathrm{H}, 2 \mathrm{NH}_{2}, \mathrm{D}_{2} \mathrm{O}\right.$ exchangeable), 5.68-5.73 (2d, 2H, pyran $\mathrm{H}-4, \mathrm{H}-5), 7.30-7.38(\mathrm{~m}, 4 \mathrm{H}$, $\left.\mathrm{C}_{6} \mathrm{H}_{4}\right), 8.26$ (s, 1H, NH, D $\mathrm{O}$ exchangeable); ${ }^{13} \mathrm{C}-\mathrm{NMR}$ $\left(\mathrm{DMSO}-d_{6}\right) \delta$ : 20.3, 22.8, 25.5, 34.8, 39.7, 116.7, 120.4, 122.6, 123.9, 124.3, 125.3, 126.9, 128.8, 130.6, 139.0, 140.9, 142.8, 144.3; MS (EI): $m / z(\%) 423\left(\mathrm{M}^{+}\right)$. Anal. Calcd for $\mathrm{C}_{22} \mathrm{H}_{19} \mathrm{ClN}_{4} \mathrm{OS}$ : C, 62.48; H, 4.53; N, 13.25; S, 7.58. Found: C, 62.22; H, 4.72; N, 13.51; S, 7.28.

6-(3-Amino-4,5,6,7-tetrahydro- $1 H$-benzo[4,5] thieno [2,3-b]pyrrol-2-yl)-4-(4-chlorophenyl)-2-hydroxy-4Hpyran-3-carbonitrile (10d)

Yellow crystals; yield: $3.21 \mathrm{~g}$ (76\%), mp: 222-223 ${ }^{\circ} \mathrm{C}$; IR $\left(\mathrm{KBr}, \mathrm{cm}^{-1}\right)$ : 3541-3333 $\left(\mathrm{NH}, \mathrm{NH}_{2}\right), 3055(\mathrm{CH}$ aromatic), $2220(\mathrm{CN}), 1626(\mathrm{C}=\mathrm{C}) ;{ }^{1} \mathrm{H}-\mathrm{NMR}$ (DMSO- $\left.d_{6}\right) \delta$ : 1.78-1.87 (m, 4H, 2CH $), 2.21-2.28\left(\mathrm{~m}, 4 \mathrm{H}, 2 \mathrm{CH}_{2}\right), 4.83$ (s, 2H, $\mathrm{NH}_{2}, \mathrm{D}_{2} \mathrm{O}$ exchangeable), 5.65-5.72 (2d, 2H, pyran $\mathrm{H}-4, \mathrm{H}-5), 7.30-7.41\left(\mathrm{~m}, 4 \mathrm{H}, \mathrm{C}_{6} \mathrm{H}_{4}\right), 8.24$ (s, 1H, NH, D $2 \mathrm{O}$ exchangeable), 10.28 (s, 1H, OH, $\mathrm{D}_{2} \mathrm{O}$ exchangeable); ${ }^{13} \mathrm{C}$ NMR (DMSO- $\left.d_{6}\right) \delta: 20.2,22.6,25.8,34.3,39.8,116.5$, 120.2, 122.6, 123.7, 123.9, 125.7, 126.9, 127.4, 130.2, 139.3, 141.3, 142.0, 142.8; MS (EI): $\mathrm{m} / \mathrm{z}(\%) 424\left(\mathrm{M}^{+}\right)$. Anal. Calcd for $\mathrm{C}_{22} \mathrm{H}_{18} \mathrm{ClN}_{3} \mathrm{O}_{2} \mathrm{~S}: \mathrm{C}, 62.33 ; \mathrm{H}, 4.28 ; \mathrm{N}$, 9.91; S, 7.56. Found: C, 62.09; H, 4.46; N, 9.75; S, 7.39.

2-Amino-6-(3-amino-4,5,6,7-tetrahydro- $1 H$-benzo $[4,5]$ thieno $[2,3-b]$ pyrrol-2-yl)-4-(4-methoxyphenyl4H-pyran-3-carbonitrile (10e)
Orange crystals; yield: $3.01 \mathrm{~g}(72 \%)$, mp: 167-168 ${ }^{\circ} \mathrm{C}$; IR $\left(\mathrm{KBr}, \mathrm{cm}^{-1}\right)$ : 3531-3312 $\left(\mathrm{NH}, \mathrm{NH}_{2}\right), 3058(\mathrm{CH}$ aromatic), $2223(\mathrm{CN}), 1628(\mathrm{C}=\mathrm{C}) ;{ }^{1} \mathrm{H}-\mathrm{NMR}$ (DMSO$\left.d_{6}\right) \delta: 1.74-1.86\left(\mathrm{~m}, 4 \mathrm{H}, 2 \mathrm{CH}_{2}\right), 2.20-2.28(\mathrm{~m}, 4 \mathrm{H}$, $\left.2 \mathrm{CH}_{2}\right), 3.01\left(\mathrm{~s}, 3 \mathrm{H}, \mathrm{OCH}_{3}\right), 4.86,5.22\left(2 \mathrm{~s}, 4 \mathrm{H}, 2 \mathrm{NH}_{2}\right.$, $\mathrm{D}_{2} \mathrm{O}$ exchangeable), 5.67-5,74 (2d, 2H, pyran $\left.\mathrm{H}-4, \mathrm{H}-5\right)$, $7.32-7.38\left(\mathrm{~m}, 4 \mathrm{H}, \mathrm{C}_{6} \mathrm{H}_{4}\right), 8.25\left(\mathrm{~s}, 1 \mathrm{H}, \mathrm{NH}, \mathrm{D}_{2} \mathrm{O}\right.$ exchangeable); ${ }^{13} \mathrm{C}-\mathrm{NMR}$ (DMSO- $\left.d_{6}\right) \delta$ : 20.0, 22.8, 25.8, 34.8, 30.8 , 39.6, 116.9, 120.6, 122.6, 123.4, 123.9, 125.7, $126.9,127.6,130.4,139.4,141.7,142.3,143.6$; MS (EI): $m / z(\%) 418\left(\mathrm{M}^{+}\right)$. Anal. Calcd for $\mathrm{C}_{23} \mathrm{H}_{22} \mathrm{~N}_{4} \mathrm{O}_{2} \mathrm{~S}: \mathrm{C}$, 66.01; H, 5.30; N, 13.39; S, 7.66. Found: C, 66.24; H, 5.48; N, 13.19; S, 7.80.

6-(3-Amino-4,5,6,7-tetrahydro- $1 H$-benzo[4,5]thieno [2,3-b]pyrrol-2-yl)-2-hydroxy-4-(4-methoxyphenyl)4H-pyran-3-carbonitrile (10f)

Orange crystals; yield: $3.01 \mathrm{~g}$ (70\%), mp: 229-230 ${ }^{\circ} \mathrm{C}$; IR $\left(\mathrm{KBr}, \mathrm{cm}^{-1}\right)$ : 3566-3332 (NH, $\left.\mathrm{NH}_{2}, \mathrm{OH}\right)$, $3056\left(\mathrm{CH}\right.$ aromatic), $2220(\mathrm{CN}), 1626(\mathrm{C}=\mathrm{C})$; ${ }^{1} \mathrm{H}-\mathrm{NMR}$ $\left(\mathrm{DMSO}-d_{6}\right) \delta$ : 1.74-1.86 (m, 4H, 2CH $\left.\mathrm{CH}_{2}\right), 2.22-2.29(\mathrm{~m}$, $\left.4 \mathrm{H}, 2 \mathrm{CH}_{2}\right), 3.08\left(\mathrm{~s}, 3 \mathrm{H}, \mathrm{OCH}_{3}\right), 4.83\left(\mathrm{~s}, 2 \mathrm{H}, \mathrm{NH}_{2}, \mathrm{D}_{2} \mathrm{O}\right.$ exchangeable), 5.64, $5.71(2 \mathrm{~d}, 2 \mathrm{H}$, pyran $\mathrm{H}-4, \mathrm{H}-5)$, 7.30-7.44 (m, 4H, $\left.\mathrm{C}_{6} \mathrm{H}_{4}\right), 8.23$ (s, 1H, NH, $\mathrm{D}_{2} \mathrm{O}$ exchangeable), 10.32 (s, 1H, D $\mathrm{O}$ exchangeable, $\mathrm{OH}) ;{ }^{13} \mathrm{C}-\mathrm{NMR}$ $\left(\mathrm{DMSO}-d_{6}\right) \delta: 20.5,22.8,25.3,34.5,30.8,39.1,116.9$, 120.6, 122.6, 123.4, 123.9, 125.7, 126.9, 127.6, 130.6, 139.4, 141.7, 142.3, 143.9; MS (EI): $m / z(\%) 419\left(\mathrm{M}^{+}\right)$. Anal. Calcd for $\mathrm{C}_{23} \mathrm{H}_{21} \mathrm{~N}_{3} \mathrm{O}_{3} \mathrm{~S}: \mathrm{C}, 65.85 ; \mathrm{H}, 5.05 ; \mathrm{N}$, 10.02; S, 7.64. Found: C, 66.19; H, 5.17; N, 10.22; S, 7.59 .

\section{1. 7. General Procedure for the Synthesis of Benzo[4,5]thieno-[2,3-b]pyrrol-2-yl)-2-(2- Cyanoacetamido)thiophene Derivatives 12a and 12b}

To a solution of either compound 8a (3.14 g, 0.01 $\mathrm{mol})$ or $\mathbf{8 b}(3.61 \mathrm{~g}, 0.01 \mathrm{~mol})$ in dimethylformamide (40 $\mathrm{mL}$ ) ethyl cyanoacetate was added. The reaction mixture was heated under reflux for $2 \mathrm{~h}$ then poured onto ice/water. The formed solid product was collected by filtration and crystallized from ethanol.

$N$-(4-(3-Amino-4,5,6,7-tetrahydro-1H-benzo[4,5]thieno [2,3-b]pyrrol-2-yl)-3-cyano-thiophen-2-yl)-1-cyanoacetamide (12a)

Yellow crystals; yield: $3.43 \mathrm{~g}$ (90\%), mp: 184-185 ${ }^{\circ} \mathrm{C}$; IR $\left(\mathrm{KBr}, \mathrm{cm}^{-1}\right)$ : 3482-3323 $\left(\mathrm{NH}, \mathrm{NH}_{2}\right), 3055(\mathrm{CH}$ aromatic), 2225, $2220(2 \mathrm{CN}), 1705(\mathrm{C}=\mathrm{O}), 1630(\mathrm{C}=\mathrm{C})$; ${ }^{1} \mathrm{H}-\mathrm{NMR}$ (DMSO- $\left.d_{6}\right) \delta$ : 1.79-1.83 (m, 4H, 2 $\left.\mathrm{CH}_{2}\right)$, 2.25-2.26 (m, 4H, 2CH $), 4.83\left(\mathrm{~s}, 2 \mathrm{H}, \mathrm{NH}_{2}, \mathrm{D}_{2} \mathrm{O}\right.$ exchangeable), $5.20\left(\mathrm{~s}, 2 \mathrm{H}, \mathrm{CH}_{2}\right), 6.20(\mathrm{~s}, 1 \mathrm{H}$, thiophene $\mathrm{H}-5)$, $8.28,8.32\left(2 \mathrm{~s}, 2 \mathrm{H}, \mathrm{NH}, \mathrm{D}_{2} \mathrm{O}\right.$ exchangeable); ${ }^{13} \mathrm{C}-\mathrm{NMR}$ $\left(\mathrm{DMSO}-d_{6}\right) \delta: 20.3,22.9,25.4,34.7,52.7,116.9,117.2$, $120.3,123.1,124.1,124.6,125.3,127.2,138.8,141.2$, 
142.6, 168.2; MS (EI): $m / z(\%) 381\left(\mathbf{M}^{+}\right)$. Anal. Calcd for $\mathrm{C}_{18} \mathrm{H}_{15} \mathrm{~N}_{5} \mathrm{OS}_{2}$ : C, 56.67; H, 3.96; N, 18.36; S, 16.81. Found: C, 56.88; H, 3.58; N, 18.56; S, 16.93.

Ethyl 4-(3-amino-4,5,6,7-tetrahydro-1H-benzo[4,5] thieno-[2,3-b]pyrrol-2-yl)-2-(2-cyano-acetamido) thiophene-3-carboxylate $(12 b)$

Orange crystals; yield: 2.99 g (70\%); mp: 194-195 ${ }^{\circ} \mathrm{C}$; IR $\left(\mathrm{KBr}, \mathrm{cm}^{-1}\right): 3453-3320\left(\mathrm{NH}, \mathrm{NH}_{2}\right), 3056(\mathrm{CH}$ aromatic), 2223, 1702, $1688(2 \mathrm{C}=\mathrm{O}), 1632(\mathrm{C}=\mathrm{C})$; ${ }^{1} \mathrm{H}-\mathrm{NMR}\left(\mathrm{DMSO}-d_{6}\right) \delta$ : $1.13\left(\mathrm{t}, 3 \mathrm{H}, J=6.83 \mathrm{~Hz}, \mathrm{CH}_{3}\right)$, 1.81-1.87 (m, 4H, 2 $\left.\mathrm{CH}_{2}\right), 2.22-2.25\left(\mathrm{~m}, 4 \mathrm{H}, 2 \mathrm{CH}_{2}\right), 4.23$ (q, 2H, $\left.J=6.83 \mathrm{~Hz}, \mathrm{CH}_{2}\right), 4.81\left(\mathrm{~s}, 2 \mathrm{H}, \mathrm{NH}_{2}, \mathrm{D}_{2} \mathrm{O}\right.$ exchangeable), $5.23\left(\mathrm{~s}, 2 \mathrm{H}, \mathrm{CH}_{2}\right), 6.23(\mathrm{~s}, 1 \mathrm{H}$, thiophene $\mathrm{H}-5)$, $8.30,8.34\left(\mathrm{~s}, 2 \mathrm{H}, 2 \mathrm{NH}, \mathrm{D}_{2} \mathrm{O}\right.$ exchangeable); ${ }^{13} \mathrm{C}-\mathrm{NMR}$ $\left(\mathrm{DMSO}-d_{6}\right) \delta: 16.0,20.3,22.2,25.6,34.8,47.1,51.4$, $116.5,120.4,122.7,123.8,124.3,124.9,127.6,133.9$, 143.8, 164.3, 170.2; MS (EI): $\mathrm{m} / z$ (\%) $428\left(\mathrm{M}^{+}\right)$. Anal. Calcd for $\mathrm{C}_{20} \mathrm{H}_{20} \mathrm{~N}_{4} \mathrm{O}_{3} \mathrm{~S}_{2}$ : C, 56.06; H, 4.70; N, 13.07; S, 14.97. Found: C, 56.22; H, 4.53; N, 13.31; S, 15.07.

\section{1. 8. General Procedure for the Synthesis of Hydrazoacetamide Derivatives 14a-d}

To a cold solution $\left(0-5{ }^{\circ} \mathrm{C}\right)$ of compound $12 \mathbf{a}(3.81$ $\mathrm{g}, 0.01 \mathrm{~mol})$ in ethanol $(50 \mathrm{~mL})$ containing sodium acetate $(3.50 \mathrm{~g}, 0.50 \mathrm{~mol})$ either benzenediazonium chloride $(0.01$ $\mathrm{mol})$, 4-chlorobenzene-diazonium chloride $(0.01 \mathrm{~mol})$, 4-methoxybenzenediazonium chloride $(0.01 \mathrm{~mol})$ or 4-methylaniline $(0.01 \mathrm{~mol})$ [prepared by adding a cold solution of sodium nitrite $(0.70 \mathrm{~g}$, in water $(10 \mathrm{~mL}))$ to a cold solution $\left(0-5{ }^{\circ} \mathrm{C}\right)$ of either aniline oil $(0.93 \mathrm{~g}, 0.01 \mathrm{~mol})$, 4-chloroaniline $(1.27 \mathrm{~g}, 0.01 \mathrm{~mol})$ 4-methoxybenzenediazonium chloride $(1.24 \mathrm{~g}, 0.01 \mathrm{~mol})$ or 4-methylaniline $(1.07 \mathrm{~g}, 0.01 \mathrm{~mol})$ in concentrated hydrochloric acid (12 $\mathrm{mL}$ ) with continuous stirring] was added with continuous stirring. The whole reaction mixture was left at room temperature for $1 \mathrm{~h}$ then the formed solid product was collected by filtration and crystallized from acetic acid.

2-((4-(3-amino-4,5,6,7-tetrahydro- $1 H$-benzo[4,5]thieno[2,3-b]pyrrol-2-yl)-3-cyanothiophen-2-yl)amino)-2oxo- $N$ '-phenylacetohydrazonoyl cyanide (14a)

Red crystals; yield: $3.78 \mathrm{~g}(78 \%)$, mp: $223-224{ }^{\circ} \mathrm{C}$; IR ( $\left.\mathrm{KBr}, \mathrm{cm}^{-1}\right)$ : 3475-3320 (NH), 3053 ( $\mathrm{CH}$ aromatic), 2223, 2220 (2CN), 1708 (C=O), $1630(\mathrm{C}=\mathrm{C})$; ${ }^{1} \mathrm{H}-\mathrm{NMR}$ $\left(\mathrm{DMSO}-d_{6}\right) \delta$ : 1.77-1.85 (m, 4H, 2CH$), 2.25-2.28(\mathrm{~m}$, $4 \mathrm{H}, 2 \mathrm{CH}_{2}$ ), 4.80 (s, $2 \mathrm{H}, \mathrm{NH}_{2}, \mathrm{D}_{2} \mathrm{O}$ exchangeable), 6.15 (s, $1 \mathrm{H}$, thiophene $\mathrm{H}-5), 7.25-7.41\left(\mathrm{~m}, 5 \mathrm{H}, \mathrm{C}_{6} \mathrm{H}_{5}\right), 8.25,8.30$, $8.56\left(3 \mathrm{~s}, 3 \mathrm{H}, 3 \mathrm{NH}, \mathrm{D}_{2} \mathrm{O}\right.$ exchangeable); ${ }^{13} \mathrm{C}-\mathrm{NMR}$ (DM$\left.\mathrm{SO}-d_{6}\right) \delta$ : 20.5, 22.9, 25.8, 34.7, 116.7, 117.0, 120.2, $121.7,123.1,124.0,124.1,124.6,125.3,126.9,127.2$, 129.3, 133.1, 138.8, 141.2, 142.8, 164.2, 168.7; MS (EI): $m / z(\%) 485\left(\mathrm{M}^{+}\right)$. Anal. Calcd for $\mathrm{C}_{24} \mathrm{H}_{19} \mathrm{~N}_{7} \mathrm{OS}_{2}$ : C, 59.36; H, 3.94; N, 20.19; S, 13.21. Found: C, 59.42; H, $3.72 ; \mathrm{N}, 20.53 ; \mathrm{S}, 13.08$.
2-((4-(3-Amino-4,5,6,7-tetrahydro-1H-benzo[4,5]thieno[2,3-b]pyrrol-2-yl)-3-cyanothiophen-2-yl)amino)- $N$ '(4-chlorophenyl)-2-oxoacetohydrazonoyl cyanide (14b) Red crystals; yield: $4.41 \mathrm{~g}$ (85\%), mp: $194-195^{\circ} \mathrm{C}$; IR $\left(\mathrm{KBr}, \mathrm{cm}^{-1}\right)$ : 3488-3315 $\left(\mathrm{NH}, \mathrm{NH}_{2}\right), 3056(\mathrm{CH}$ aromatic), 2225, 2220 (2CN), $1710(\mathrm{C}=\mathrm{O}), 1628(\mathrm{C}=\mathrm{C})$; ${ }^{1} \mathrm{H}-\mathrm{NMR}$ $\left(\mathrm{DMSO}-d_{6}\right) \delta: 1.79-1.85\left(\mathrm{~m}, 4 \mathrm{H}, 2 \mathrm{CH}_{2}\right), 2.23-2.27(\mathrm{~m}, 4 \mathrm{H}$, $\left.2 \mathrm{CH}_{2}\right), 4.83\left(\mathrm{~s}, 2 \mathrm{H}, \mathrm{NH}_{2}, \mathrm{D}_{2} \mathrm{O}\right.$ exchangeable), $6.12(\mathrm{~s}, 1 \mathrm{H}$, thiophene $\mathrm{H}-5), 7.28-7.39\left(\mathrm{~m}, 4 \mathrm{H}, \mathrm{C}_{6} \mathrm{H}_{4}\right), 8.23,8.32,8.42$ (3s, $3 \mathrm{H}, 3 \mathrm{NH}, \mathrm{D}_{2} \mathrm{O}$ exchangeable); ${ }^{13} \mathrm{C}-\mathrm{NMR}$ (DMSO- $\left.d_{6}\right) \delta$ : 20.6, 22.4, 25.8, 34.9, 116.8, 117.3, 120.0, 121.4, 123.1, $124.0,124.1,124.8,125.3,127.2,138.8,140.4,141.2$, 143.4, 164.8, 168.6; MS (EI): $\mathrm{m} / z$ (\%) $520\left(\mathrm{M}^{+}\right)$. Anal. Calcd for $\mathrm{C}_{24} \mathrm{H}_{18} \mathrm{ClN}_{7} \mathrm{OS}_{2}: \mathrm{C}, 55.43 ; \mathrm{H}, 3.49 ; \mathrm{N}, 18.85 ; \mathrm{S}$, 12.33. Found: C, 55.70; H, 3.62; N, 18.59; S, 12.48 .

2-((4-(3-Amino-4,5,6,7-tetrahydro-1H-benzo[4,5]thieno[2,3-b]pyrrol-2-yl)-3-cyanothiophen-2-yl)amino)- $N$ '(4-methoxyphenyl)-2-oxoacetohydrazonoyl cyanide (14c) Reddish brown crystals; yield: $4.63 \mathrm{~g}$ (90\%); mp: 168-169 ${ }^{\circ} \mathrm{C}$; IR $\left(\mathrm{KBr}, \mathrm{cm}^{-1}\right)$ : 3462-3335 (NH, $\left.\mathrm{NH}_{2}\right), 3053$ (CH aromatic), 2227, $2221(2 \mathrm{CN}), 1720(\mathrm{C}=\mathrm{O}), 1638$ $(\mathrm{C}=\mathrm{C}) ;{ }^{1} \mathrm{H}-\mathrm{NMR}\left(\mathrm{DMSO}-d_{6}\right) \delta: 1.74-1.82\left(\mathrm{~m}, 4 \mathrm{H}, 2 \mathrm{CH}_{2}\right)$, 2.21-2.28 (m, 4H, 2CH $\mathrm{CH}_{2}, 3.38\left(\mathrm{~s}, 3 \mathrm{H}, \mathrm{OCH}_{3}\right), 4.88(\mathrm{~s}$, $2 \mathrm{H}, \mathrm{NH}_{2}, \mathrm{D}_{2} \mathrm{O}$ exchangeable), $6.13(\mathrm{~s}, 1 \mathrm{H}$, thiophene $\mathrm{H}-5), 7.31-7.42\left(\mathrm{~m}, 4 \mathrm{H}, \mathrm{C}_{6} \mathrm{H}_{4}\right), 8.21,8.32,8.45(3 \mathrm{~s}, 3 \mathrm{H}$, $3 \mathrm{NH}, \mathrm{D}_{2} \mathrm{O}$ exchangeable); ${ }^{13} \mathrm{C}-\mathrm{NMR}$ (DMSO- $d_{6}$ ) $\delta: 20.8$, 22.7, 25.8, 34.3, 55.3, 116.3, 117.0, 120.3, 121.4, 123.8, $124.0,124.0,124.8,125.9,127.0,133.2,138.2,140.8$, 141.9, 164.9, 168.6; MS (EI): $\mathrm{m} / \mathrm{z}(\%) 516\left(\mathrm{M}^{+}\right)$. Anal. Calcd for $\mathrm{C}_{25} \mathrm{H}_{21} \mathrm{~N}_{7} \mathrm{O}_{2} \mathrm{~S}_{2}: \mathrm{C}, 58.24 ; \mathrm{H}, 4.11 ; \mathrm{N}, 19.02 ; \mathrm{S}$, 12.44. Found: C, 58.40; H, 4.26; N, 19.11; S, 12.29.

2-((4-(3-Amino-4,5,6,7-tetrahydro- $1 H$-benzo[4,5] thieno[2,3-b]pyrrol-2-yl)-3-cyanothiophen-2-yl)amino)-2oxo- $N^{\prime}$-(p-tolyl)acetohydrazonoyl cyanide (14d)

Reddish brown crystals; yield: $3.44 \mathrm{~g}$ (69\%); mp: 129-130 ${ }^{\circ} \mathrm{C}$; IR (KBr, cm $\left.{ }^{-1}\right)$ : 3482-3318 (NH, $\left.\mathrm{NH}_{2}\right), 3057$ $(\mathrm{CH}$ aromatic), 2227, $2220(2 \mathrm{CN}), 1712(\mathrm{C}=\mathrm{O}), 1630$ $(\mathrm{C}=\mathrm{C}) ;{ }^{1} \mathrm{H}-\mathrm{NMR}\left(\mathrm{DMSO}-d_{6}\right) \delta: 1.76-1.83\left(\mathrm{~m}, 4 \mathrm{H}, 2 \mathrm{CH}_{2}\right)$, 2.23-2.28 (m, 4H, 2CH $\left.\mathrm{CH}_{2}\right), 2.65\left(\mathrm{~s}, 3 \mathrm{H}, \mathrm{CH}_{3}\right), 4.86(\mathrm{~s}, 2 \mathrm{H}$, $\mathrm{NH}_{2}, \mathrm{D}_{2} \mathrm{O}$ exchangeable), $6.11(\mathrm{~s}, 1 \mathrm{H}$, thiophene $\mathrm{H}-5)$, 7.30-7.39 (m, 4H, $\left.\mathrm{C}_{6} \mathrm{H}_{4}\right), 8.23,8.30,8.48(3 \mathrm{~s}, 3 \mathrm{H}, 3 \mathrm{NH}$, $\mathrm{D}_{2} \mathrm{O}$ exchangeable); ${ }^{13} \mathrm{C}-\mathrm{NMR}$ (DMSO- $d_{6}$ ) $\delta: 20.4,22.9$, 23.3, 25.8, 34.6, 116.4, 117.3, 120.6, 122.8, 123.8, 124.0, $124.3,124.8,125.2,126.4,138.8,140.6,141.7,143.9$, 164.6, 168.7; MS (EI): $\mathrm{m} / z(\%) 500\left(\mathrm{M}^{+}\right)$. Anal. Calcd for $\mathrm{C}_{25} \mathrm{H}_{21} \mathrm{~N}_{7} \mathrm{OS}_{2}$ : C, 60.10; H, 4.24; N, 19.62; S, 12.84. Found: C, 60.32; H, 4.52; N, 19.48; S, 12.64.

\section{1. 9. General Procedure for the Synthesis of Thieno[2,3-b]pyridine Derivatives 15a and 15b}

A suspension of either compound 12a $(3.81 \mathrm{~g}, 0.01$ $\mathrm{mol})$ or $\mathbf{1 2 b}(4.28 \mathrm{~g}, 0.01 \mathrm{~mol})$ in sodium ethoxide $(0.02$ 
mol) [prepared by dissolving metallic sodium $(0.46 \mathrm{~g}$, $0.02 \mathrm{~mol})$ in absolute ethanol $(20 \mathrm{~mL}]$ was heated in a boiling water bath for $12 \mathrm{~h}$ then poured onto ice/water containing few drops of hydrochloric acid. The formed solid product was collected by filtration and crystallized from 1,4-dioxane.

\section{4-Amino-3-(3-amino-4,5,6,7-tetrahydro-1H-benzo[4,5] thieno[2,3-b]pyrrol-2-yl)-6-hydroxy-thieno[2,3-b]pyri dine-5-carbonitrile (15a)}

Yellow crystals; yield: $2.29 \mathrm{~g}(60 \%)$; mp: $>300{ }^{\circ} \mathrm{C}$; IR $\left(\mathrm{KBr}, \mathrm{cm}^{-1}\right)$ : 3593-3355 (NH, $\left.\mathrm{NH}_{2}, \mathrm{OH}\right), 3056(\mathrm{CH}$ aromatic), $2224(\mathrm{CN}), 1635(\mathrm{C}=\mathrm{C}) ;{ }^{1} \mathrm{H}-\mathrm{NMR}\left(\mathrm{DMSO}-d_{6}\right)$ $\delta: 1.75-1.85\left(\mathrm{~m}, 4 \mathrm{H}, 2 \mathrm{CH}_{2}\right), 2.23-2.27\left(\mathrm{~m}, 4 \mathrm{H}, 2 \mathrm{CH}_{2}\right)$, 4.68, $5.09\left(2 \mathrm{~s}, 4 \mathrm{H}, 2 \mathrm{NH}_{2}, \mathrm{D}_{2} \mathrm{O}\right.$ exchangeable), $6.16(\mathrm{~s}, 1 \mathrm{H}$, thiophene $\mathrm{H}-5), 8.28\left(\mathrm{~s}, 1 \mathrm{H}, \mathrm{NH}, \mathrm{D}_{2} \mathrm{O}\right.$ exchangeable), 9.90 (s, $1 \mathrm{H}, \mathrm{OH}, \mathrm{D}_{2} \mathrm{O}$ exchangeable); ${ }^{13} \mathrm{C}-\mathrm{NMR}$ (DMSO$\left.d_{6}\right) \delta: 20.8,22.9,25.8,34.7,116.7,120.2,121.7,123.1$, $124.1,124.6,125.3,126.5,127.0,129.6,138.8,142.8$, 144.5, 162.8; MS (EI): $\mathrm{m} / z(\%) 381\left(\mathrm{M}^{+}\right)$. Anal. Calcd for $\mathrm{C}_{18} \mathrm{H}_{15} \mathrm{~N}_{5} \mathrm{OS}_{2}$ : C, 56.67; H, 3.96; N, 18.36; S, 16.81 . Found: C, 56.93; H, 3.65; N, 18.48; S, 17.09.

\section{3-(3-Amino-4,5,6,7-tetrahydro- $1 H$-benzo-4,5] thieno [2,3-b]pyrrol-2-yl)-4,6-dihydroxy-thieno $[2,3-b]$ pyridi- ne-5-carbonitrile (15b)}

Yellow crystals; yield: $2.79 \mathrm{~g}(73 \%) \mathrm{g})$; $\mathrm{mp}$ : 289-290 ${ }^{\circ} \mathrm{C}$; IR $\left(\mathrm{KBr}, \mathrm{cm}^{-1}\right)$ : 3578-3345 $\left(\mathrm{NH}, \mathrm{NH}_{2}, \mathrm{OH}\right)$, $3056\left(\mathrm{CH}\right.$ aromatic), $2222(\mathrm{CN}), 1628(\mathrm{C}=\mathrm{C}) ;{ }^{1} \mathrm{H}-\mathrm{NMR}$ (DMSO- $\left.d_{6}\right) \delta: 1.79-1.85\left(\mathrm{~m}, 4 \mathrm{H}, 2 \mathrm{CH}_{2}\right), 2.23-2.27(\mathrm{~m}$, $4 \mathrm{H}, 2 \mathrm{CH}_{2}$ ), 4.86 (s, $2 \mathrm{H}, \mathrm{NH}_{2}, \mathrm{D}_{2} \mathrm{O}$ exchangeable), 6.17 (s, $1 \mathrm{H}$, thiophene $\mathrm{H}-5), 8.26\left(\mathrm{~s}, 1 \mathrm{H}, \mathrm{NH}, \mathrm{D}_{2} \mathrm{O}\right.$ exchangeable), $10.29, \quad 10.34\left(2 \mathrm{~s}, 2 \mathrm{H}, \quad \mathrm{D}_{2} \mathrm{O}\right.$ exchangeable, 2OH); ${ }^{13} \mathrm{C}$-NMR (DMSO- $d_{6}$ ) $\delta: 20.3,22.8,25.8,34.7,116.6$, 120.2, 121.6, 123.1, 124.7, 124.1, 124.8, 125.3, 126.8, 127.5, 133.2, 140.8, 143.8, 144.2, 162.9; MS (EI): $\mathrm{m} / \mathrm{z}$ (\%) $382\left(\mathrm{M}^{+}\right)$. Anal. Calcd for $\mathrm{C}_{18} \mathrm{H}_{14} \mathrm{~N}_{4} \mathrm{O}_{2} \mathrm{~S}_{2}: \mathrm{C}, 56.53 ; \mathrm{H}$, 3.69 ; N, 14.65; S, 16.77. Found: C, 56.72; H, 3.46; N, $14.80 ; \mathrm{S}, 16.37$.

\section{Conclusions}

Novel 4,5,6,7-tetrahydro-1 $H$-benzo[4,5]thieno[2,3$b$ ]pyrrol-derivatives were synthesized in good yields. Some compounds were used to produce annulated products. The cytotoxicity of the newly synthesized compounds indicate that compounds $4,7 a, 7 b, 8 a, 8 b, 10 c, 10 d, 10 f$, 12a, 12b, 14b and $15 b$ showed the highest potency among the tested compounds. In addition, the anti- proliferative evaluations of these twelve compounds indicated that the benzo[4',5'] thieno[ $\left.3^{\prime}, 2^{\prime}: 4,5\right]$ pyrrolo[3,2- $\left.b\right]$ pyridine derivative $\mathbf{7 b}$ and the benzo[4,5]thieno-[2,3-b]pyrrol-2-yl)thiophene derivative $\mathbf{8 b}$ showed high potency against Molt4/C8 and CEM cell lines and their IC $_{50}$ 's are higher than the reference drug "doxorubicin".

\section{Acknowledgments}

R. M. Mohareb would like to thank the Alexander von Humboldt for affording him regular fellowships in Germany for doing research and completing this work.

\section{References}

1. S. Xue, H. Guo, M. Liu, J. Jin, D. Ju, Z. Liu, Z. Li, Eur. J. Med. Chem. 2015, 26, 151-161.

http://dx.doi.org/10.1016/j.ejmech.2015.04.016

2. L. Ye, J. He, Z. Hu, Q. Dong, H. Wang, F. Fu, J. Tian, Food Chem. Toxicol. 2013, 52, 200-206.

http://dx.doi.org/10.1016/j.fct.2012.11.004

3. S. Hama, S. Utsumi, Y. Fukuda, K. Nakayama, Y. Okamura, H. Tsuchiya, K. Fukuzawa, H. Harashima, K. Kogure, J. Controlled Release. 2012, 161, 843-851.

http:// 10.1016/j.jconrel.2012.05.031

4. I. M. Fakhr, M. A. Radwan, S. El-Batran, O. M. Abd El-Salam, S. M. El-Shenawy, Eur. J. Med. Chem. 2009, 44, 17181725. http:// 10.1016/j.ejmech.2008.02.034

5. S. Bondock, W. Fadaly, M. A. Metwally, Eur. J. Med. Chem. 2010, 45, 3692-3701.

http://dx.doi.org/10.1016/j.ejmech.2010.05.018

6. C. K. Ryu, S. K. Lee, J. Y. Han, O. J. Jung, J. Y. Lee, S. H. Jeong, Bioorg. Med. Chem. Lett. 2005, 15, 2617-2620.

http://doi.org/10.1016/j.bmcl.2005.03.042

7. L. J. Gonzalez, C. E. Stephens, T. Wenzler, R. Brun, F. A. Tanious, W. D. Wilson, T. Barszcz, K. A. Werbovetz, D. W. Boykin, Eur. J. Med. Chem. 2007, 42, 552-557. http://dx.doi.org/10.1016/j.ejmech.2006.11.006

8. J. B. Hudson, E. A. Graham, N. Miki, G. H. N. Towers, L. L. Hudson, R. Rossi, A. Carpita, D. Neri, Chemosphere. 1989, 19, 1329-1343. http://dx.doi.10.1016/0045-6535(89)90080-5

9. K. I. Molvi, K. K. Vasu, S. G. Yerande, V. Sudarsanam, N. Haque, Eur. J. Med. Chem. 2007, 42, 1049-1058. http://dx.doi.10.1016/j.ejmech.2007.01.007

10. R. Kulandasamy, A. V. Adhikari, J. P. Stables, Eur. J. Med. Chem. 2009, 44, 4376-4384.

http://dx.doi.10.1016/j.ejmech. 2009.05.026

11. H. J. Jung, Y. S. Song, C. J. Lim, E. H. Park, J. Ethanopharmacol. 2009, 126, 355-360.

http://dx.doi.10.1016/j.jep.2009.08.031

12. W. O. Chi, L. H. Chang, Inorg. Chim. Acta. 1986, 125, 203-206. http://dx.doi. 10.1016/S0020-1693(00)81212-8.

13. D. C. S. Friedman, P. Friedman, J. Mol. Struct. Theochem, 1995, 333, 71-78. http://dx.doi. 10.1016/0166-1280(94)03930-J

14. M. K. Parai, G. Panda, V. Chaturvedi, Y. K. Manju, S. Sinha, Bioorg. Med. Chem. Lett. 2008, 18, 289-292. http://dx.doi. 10.1016/j.bmcl.2007.10.083

15. D. Caridha, A. K. Kathcart, D. Jirage, N. C. Waters, Bioorg. Med. Chem. Lett. 2010, 20, 3863-3867.

http://dx.doi.org/10.1016/j.bmcl.2010.05.039 
16. G. Fear, S. Komarnytsky, I. Raskin, Pharm. Therap. 2007 , 113, 354-368. http://dx.doi. 10.1016/j.pharmthera.2006.09.001

17. B. O. Al-Najjar, H. A. Wahab, T. S. T. Muhammad, A. C. Shu-Chien, N. A. A. Noruddin, M. O. Taha, Eur. J. Med. Chem. 2011, 46, 2513-2529.

http://dx.doi.org/10.1016/j.bmcl.2010.05.039

18. R. M Mohareb, N. S. Abbas, A. R. Ibrahim, Acta Chim. Solv. 2013, 60, 583-594. http://dx.doi.org/ 0.17344/acsi.2013.1418

19. R. M. Mohareb, E. Z. El-Arab, K. A. El-Sharkawy, Sci. Pharm. 2009, 77, 355-366. http://dx.doi.org/10.3797/scipharm.0901-20

20. R. M. Mohareb, N. Y. Megally Abdo, Molecules. 2015, 20, 11535-11553. doi:10.3390/molecules200611535

21. R. M. Mohareb, N. Y. Megally Abdo, Chem. Pharm. Bull. 2015, 63, 678-687. http://dx.doi.org/10.1248/cpb.c15-00115

22. R. M. Mohareb, M. Y. Zaki, N. S. Abbas, Steroids. 2015, 98 , 80-91. http://dx.doi. 10.1016/j.steroids.2015.03.001

23. R. M. Mohareb, F. Al-Omran, Steroids. 2012, 77, 15511559. http://dx.doi.org/10.1016/j.steroids.2012.09.007

24. B. P. McKibben, C. H. Cartwright, A. L. Castelhano, Tetrahedron Lett. 1999, 40, 5471-5474.

http://dx.doi.org/10.1016/S0040-4039(99)01108-9
25. K. Balamurugan, S. Perumal, S. K. Reddy, P. Yogeeswari, D. Sriram, Tetrahedron Lett. 2009, 50, 6191-6195. http://dx.doi.org/10.1016/j.tetlet.2009.08.085

26. R. M. Mohareb, A. E. Mahmoud, M. A. Abdelaziz, Med. Chem. Res. 2014, 23, 564-579. http://dx.doi.org/10.1007/s00044-013-0664

27. D. Briel, A. Rybak, C. Kronbach, K. Unverferth, Eur. J. Med. Chem. 2010, 45, 69-77. http://dx.doi.org/10.1016/j.ejmech.2009.09.025

28. A. Monks, D. Scudiero, P. Skehan, R. Shoemaker, K. Paull, D. Vistica, C. Hose, J. Langley, P. Cronise, A. Waigro-Wolff, M. Gray-Goodrich, H. Campbell, J. Mayo, M. Boyd, J. Natl. Cancer Inst. 1991, 83, 757-766. http://dx.doi.org/10.1093/jnci/83.11.757

29. K. D. Paull, H. Shoemaker, L. Hodes, A. Monks, D. A. Scudiero, L. Rubinstein, J. Plowman, M. R. Boyd, J. Natl. Cancer Inst. 1989, 81, 1088-1092. http://dx.doi.org/10.1093/jnci/81.14.1088

30. L. S. Olsen, P. J. Hjarnaa, S. Latini, P. K. Holm, R. Larsson, E. Bramm, L.Binderup, M. W. Madsen, Int. J. Cancer. 2004, 111, 198-205. http://dx.doi.org/10.1002/ijc. 20255

31. A. Svensson, U. Bäckman, E. Jonsson, R. Larsson, R. Christofferson, Pediatr Res. 2002, 51, 607-611. http://dx.doi.org/10.1203/00006450-200205000

\section{Povzetek}

Iz 2-amino-3-ciano-4,5,6,7-tetrahidrobenzo[b]tiofena (1) smo z reakcijo z with $\alpha$-kloroacetonom sintetizrali $N$-alkil derivat (3), tetrahidrobenzo[b]tienopirol. Spojino 3 smo v raztopini natrijevega etoksida s ciklizacijo pretvorili v tetrahidrobenzo[b]tienopirol (4), ki smo ga uporabili naprej za sinteze derivatov tiofena, tieno[2,3- $b]$ piridina in pirana. Citotoksičnost sintetiziranih spojin smo preverili na rakavih celicah želodčnega (NUGC), črevesnega (DLD-1), jetrnega (HA22T in HEPG-2) ter nazofaringealnega karcinoma (HONE-1), raka dojk (MCF-7) in na normalnih fibroblastnih celicah (WI-38). Izkazalo se je, da imajo spojine 4, 7a, 7b, 8a, 8b, 10c, 10d, 10f, 12a, 12b, 14b in 15b optimalni citotoksični učinek na rakave celice. Spojini $\mathbf{7 b}$ in $\mathbf{1 4 b}$ kažeta maksimalni inhibicijski efekt, ki je precej večji od efekta referenčne spojine CHS-828 (piridil cianogvanidina). 